\title{
The evolution of young HII regions
}

\section{Continuum emission and internal dynamics $\star$}

\author{
P. D. Klaassen ${ }^{1}$, K. G. Johnston ${ }^{2}$, J. S. Urquhart ${ }^{3}$, J. C. Mottram ${ }^{4}$, T. Peters ${ }^{5}$, \\ R. Kuiper ${ }^{6,4}$, H. Beuther ${ }^{4}$, F.F.S. van der Tak ${ }^{7,8}$, and C. Goddi ${ }^{9,10}$ \\ ${ }^{1}$ UK Astronomy Technology Centre, Royal Observatory Edinburgh, Blackford Hill, Edinburgh EH9 3HJ, UK \\ e-mail: pamela.klaassen@stfc.ac.uk \\ ${ }^{2}$ School of Physics \& Astronomy, E.C. Stoner Building, The University of Leeds, Leeds LS2 9JT, UK \\ ${ }^{3}$ Centre for Astrophysics and Planetary Science, University of Kent, Canterbury CT2 7NH, UK \\ ${ }^{4}$ Max Planck Institute for Astronomy, Königstuhl 17, 69117 Heidelberg, Germany \\ ${ }^{5}$ Max-Planck-Institut für Astrophysik, Karl-Schwarzschild-Str. 1, 85748 Garching, Germany \\ ${ }^{6}$ Institute of Astronomy and Astrophysics, University of Tübingen, Auf der Morgenstelle 10, 72076 Tübingen, Germany \\ ${ }^{7}$ SRON Netherlands Institute for Space Research, Landleven 12, 9747 AD Groningen, The Netherlands \\ ${ }^{8}$ Kapteyn Astronomical Institute, University of Groningen, Groningen, The Netherlands \\ 9 Department of Astrophysics/IMAPP, Radboud University, PO Box 9010, 6500 GL Nijmegen, The Netherlands \\ ${ }^{10}$ ALLEGRO/Leiden Observatory, Leiden University, PO Box 9513, 2300 RA Leiden, The Netherlands
}

Received 7 August 2017 / Accepted 30 November 2017

\begin{abstract}
Context. High-mass stars form in much richer environments than those associated with isolated low-mass stars, and once they reach a certain mass, produce ionised (HII) regions. The formation of these pockets of ionised gas are unique to the formation of high-mass stars $\left(M>8 M_{\odot}\right)$, and present an excellent opportunity to study the final stages of accretion, which could include accretion through the HII region itself.

Aims. This study of the dynamics of the gas on both sides of these ionisation boundaries in very young HII regions aims to quantify the relationship between the HII regions and their immediate environments.

Methods. We present high-resolution ( 0.5") ALMA observations of nine HII regions selected from the red MSX source survey with compact radio emission and bolometric luminosities greater than $10^{4} L_{\odot}$. We focus on the initial presentation of the data, including initial results from the radio recombination line $\mathrm{H} 29 \alpha$, some complementary molecules, and the $256 \mathrm{GHz}$ continuum emission.

Results. Of the six (out of nine) regions with $\mathrm{H} 29 \alpha$ detections, two appear to have cometary morphologies with velocity gradients across them, and two appear more spherical with velocity gradients suggestive of infalling ionised gas. The remaining two were either observed at low resolution or had signals that were too weak to draw robust conclusions. We also present a description of the interactions between the ionised and molecular gas (as traced by CS $(J=5-4)$ ), often (but not always) finding the HII region had cleared its immediate vicinity of molecules.

Conclusions. Of our sample of nine, the observations of the two clusters expected to have the youngest HII regions (from previous radio observations) are suggestive of having infalling motions in the $\mathrm{H} 29 \alpha$ emission, which could be indicative of late stage accretion onto the stars despite the presence of an HII region.
\end{abstract}

Key words. stars: massive - stars: formation - HII regions - ISM: kinematics and dynamics - submillimetre: ISM

\section{Introduction}

High-mass stars are generally observed in external galaxies because they burn brighter and hotter than their low-mass counterparts. From their light we extrapolate an entire initial mass function worth of stellar mass in those galaxies (Kennicutt \& Evans 2012). Massive (proto)stars are expected to provide strong feedback on the formation process with their high UV radiation field. When the (proto)star becomes massive enough, it begins to heat up, forming an HII region, which ionises the ambient medium, the upper and lower layers of the accretion disk, and sometimes even the outflow (e.g. Keto \& Wood 2006). The

\footnotetext{
${ }^{\star}$ Table A.2 is also available at the CDS via anonymous ftp to cdsarc.u-strasbg.fr $(130.79 .128 .5)$ or via http://cdsarc.u-strasbg.fr/viz-bin/qcat?]/A+A/611/A99
}

early evolution of hyper- and ultra-compact HII regions (HC HII and UC HII, respectively) critically depends on the pre-mainsequence evolution of the embedded massive protostar, including the so-called bloated phase (e.g. Hosokawa et al. 2010), its epoch of disk formation, and accretion rate (e.g. Kuiper \& Yorke 2013; Kuiper et al. 2016). In the bloated phase the massive protostar has a size of 10-100 times its zero age main sequence radius (Hosokawa et al. 2010), which lowers the surface temperature and consequently, the ionising radiation. At the same time, the accretion luminosity and rate are both predicted to be highly variable. These properties have an important and observable impact on the subsequent evolution of the young HII region (e.g. Klassen et al. 2012). Thus detailed observations of the early evolution of ultra-compact HII regions can provide key constraints on the pre-main-sequence evolution of high-mass stars (e.g. De Pree et al. 2014, 2015). 
Table 1. Source locations and beam properties.

\begin{tabular}{|c|c|c|c|c|c|c|c|}
\hline \multicolumn{4}{|c|}{ Source properties } & \multicolumn{4}{|c|}{ Observation properties } \\
\hline \multirow[t]{2}{*}{ Name } & \multicolumn{2}{|c|}{ Pointing centre } & \multirow{2}{*}{$\begin{array}{c}\mathrm{V}_{\mathrm{LSR}} \\
\left(\mathrm{km} \mathrm{s}^{-1}\right)\end{array}$} & \multicolumn{3}{|c|}{ Synthesised beam } & \multirow{2}{*}{$\begin{array}{l}\text { Continuum } \\
\text { rms noise } \\
\left(\mathrm{mJy} \mathrm{beam}^{-1}\right)\end{array}$} \\
\hline & $\begin{array}{c}\text { RA } \\
\text { (h:m:s) }\end{array}$ & $\begin{array}{r}\text { DEC } \\
\text { (d:m:s) }\end{array}$ & & $\begin{array}{c}\text { Bmaj } \\
\left({ }^{\prime \prime}\right)\end{array}$ & $\begin{array}{c}\mathrm{Bmin} \\
\left({ }^{\prime \prime}\right)\end{array}$ & $\begin{array}{r}\text { BPA } \\
\left({ }^{\circ}\right)\end{array}$ & \\
\hline \multicolumn{8}{|c|}{ Targets near G305 (3 regions) } \\
\hline G302.02+00.25 & $12: 43: 31.52$ & $-62: 36: 13.6$ & -37.1 & 0.61 & 0.58 & 28.38 & 0.15 \\
\hline G302.48-00.03 & $12: 47: 31.75$ & $-62: 53: 59.4$ & -37.1 & 0.61 & 0.57 & -174.51 & 0.17 \\
\hline G309.89+00.40 & $13: 50: 35.54$ & $-61: 40: 21.4$ & -57.6 & 0.69 & 0.62 & 6.17 & 0.15 \\
\hline \multicolumn{8}{|c|}{ Targets near G335 (6 regions) } \\
\hline G330.28+00.49 & $16: 03: 43.28$ & $-51: 51: 45.6$ & -93.5 & 0.71 & 0.53 & 87.19 & 0.13 \\
\hline G332.77-00.01 & $16: 17: 31.13$ & $-50: 32: 35.7$ & -95.6 & 0.62 & 0.46 & 87.62 & 0.18 \\
\hline G336.98-00.18 & $16: 36: 12.42$ & $-47: 37: 57.5$ & -75.1 & 0.63 & 0.47 & -90.00 & 0.13 \\
\hline G337.63-00.08 & $16: 38: 19.02$ & $-47: 4: 50.7$ & -56.5 & 0.61 & 0.46 & 90.00 & 0.12 \\
\hline G337.84-00.37 & $16: 40: 26.68$ & $-47: 7: 13.1$ & -40.4 & 0.62 & 0.47 & -89.07 & 0.18 \\
\hline G339.11+00.15 & $16: 42: 59.58$ & $-45: 49: 43.6$ & -78.2 & 1.68 & 1.11 & -84.79 & 0.51 \\
\hline
\end{tabular}

Forming high-mass stars produce strong stellar winds from an early age, likely altering the course of their accretion flows in the process (see, for example, Puls et al. 2008). When a forming massive protostar begins producing a higher ionising photon flux than can be absorbed by its immediate surroundings, an HII region forms and expands outwards until the ionisation and recombination balance (i.e. it reaches the Strömgren radius). For most high-mass stars $\left(M_{*} \sim 10-30 M_{\odot}\right)$, accretion will have halted before an HII region can expand outwards (Davies et al. 2011) because within this mass range, the protostar is likely to bloat as material is accreted, delaying the onset of an HII region (e.g. Hosokawa et al. 2010; Kuiper \& Yorke 2013; Haemmerlé \& Peters 2016). Beyond this threshold of $\sim 30 M_{\odot}$, the star can contract back to a main-sequence configuration even for high rates of ongoing accretion. Stars with masses much greater than this (e.g. $>60 M_{\odot}$ ) exist in our Galaxy, therefore something must allow for accretion onto the forming high-mass star in the presence of an HII region.

Very little is known about how that accretion continues, thus, further observational constraints are required. Such observations should include the dynamics of both the ionised and molecular gas components of very young (i.e. HC-) HII regions. How is the ionised gas moving? Is it contracting or expanding? Is it rotating and or infalling? How is the molecular gas moving in comparison? Could it be feeding (i.e. providing a collapsing mass reservoir) the ionised gas component? These types of questions can only be answered using comprehensive (i.e. matched sensitivity and resolution) observations of a large number of these young HII regions.

Here we present the first results of such a survey, including the largest sample of high-resolution observations of small (i.e. hyper-compact, <0.01 pc) HII regions observed so far, which can be used to quantify the dynamics of both the ionised and molecular gas. From this coherent dataset we can begin to draw conclusions about the nature of the interaction between HII regions and their environments.

Our sample is drawn from HII regions in the southern sky with known submm continuum fluxes and high-resolution radio (5 GHz) continuum images that constrain the size of the HII region. With our new ALMA observations we can trace the dynamics of the ionised gas to quantify its expansion into the ambient cloud. By understanding the dynamics of the molecular gas into which it is expanding, we determine what effects the HII region has on its environment, whether the original infall/rotation of the gas is disrupted, and how much energy the HII region feeds back into its surroundings.

Below, in Sect. 2, we present our source selection criteria and briefly outline our data reduction methods. In Sect. 3 we present our analysis of the clustering and the masses of the cores in each region, and the dynamics of both the ionised and molecular gas species emitting on small scales. We then compare the observed ionised gas velocity structures to those predicted in models. We summarise our findings in Sect. 4. Additional details concerning the combination of the multi-configuration ALMA data can be found in Appendices A and B we give a detailed description of the XCLASS modelling required to separate the overlapping ionised and molecular emission in our $\mathrm{H} 29 \alpha$ spectral window.

\section{Observations}

The red MSX source (RMS) survey has identified $\sim 600$ HII regions distributed throughout the Galactic plane (Lumsden et al. 2013). This sample of HII regions is the largest and most well characterised yet compiled, providing reliable distances (Urquhart et al. 2013), bolometric luminosities derived from spectral energy distribution (SED) modelling (Mottram et al. 2011b), and targeted high-resolution $\left(\sim 1^{\prime \prime}\right)$ radio continuum observations made with the ATCA and VLA (Urquhart et al. 2007, 2009).

From this sample we selected HII regions that are located in the southern Galactic plane, are associated with compact radio emission (radius $<5^{\prime \prime}$ ), have bolometric luminosities greater than $10^{4} L_{\odot}$, and are located between 3 and $5.5 \mathrm{kpc}$ to keep the linear scales roughly consistent. We used the ATLASGAL submm emission maps to refine our sample by selecting sources associated with massive and small dense clumps $\left(<35^{\prime \prime}\right.$; Urquhart et al. 2014).

Our selection criteria provide a distance limited sample of nine luminous $\mathrm{HC}$ and UC HII regions that are still embedded in their natal environment. In Table 1 we show the positions, distances to, and luminosities of our sample. These sources are ideally located for ALMA and the compactness of these sources means they can be observed with a single pointing with ALMA Band 6 observations ( $250 \mathrm{GHz}$, or a wavelength of $1.2 \mathrm{~mm})$. 
P. D. Klaassen et al.: The evolution of young Hil regions. I.

Table 2. Summary of radio properties of the HII regions and their host molecular clumps.

\begin{tabular}{|c|c|c|c|c|c|c|c|c|}
\hline Source name & $\begin{array}{c}\text { RA } \\
(\mathrm{J} 2000)\end{array}$ & $\begin{array}{c}\text { Dec } \\
(\mathrm{J} 2000) \\
\end{array}$ & $\begin{array}{c}\text { Distance } \\
(\mathrm{kpc})\end{array}$ & $\begin{array}{c}\log \left[M_{\text {clump }}\right] \\
\left(M_{\odot}\right)\end{array}$ & $\begin{array}{c}\log \left[L_{\mathrm{Bol}}\right] \\
\left(L_{\odot}\right)\end{array}$ & $\begin{array}{c}\text { Radio Size } \\
\left({ }^{\prime \prime}\right)\end{array}$ & $\begin{array}{l}\text { Flux }^{a} 6 \mathrm{~cm} \\
(\mathrm{mJy})\end{array}$ & $\alpha$ \\
\hline $\mathrm{G} 302.02+00.25$ & $12: 43: 31.49$ & $-62: 36: 13.7$ & $4.26 \pm 0.98$ & 2.76 & 4.16 & 2.24 & 59.5 & -0.03 \\
\hline G302.49-00.03 & $12: 47: 31.76$ & $-62: 53: 59.6$ & $3.39 \pm 0.40$ & 2.61 & 3.75 & 2.07 & 23.4 & 0.03 \\
\hline G309.89+00.40 & $13: 50: 35.54$ & $-61: 40: 21.4$ & $5.41 \pm 1.27$ & 3.12 & 4.40 & 1.79 & 1.2 & $\ldots$ \\
\hline G330.28+00.49 & $16: 03: 43.26$ & $-51: 51: 45.9$ & $5.45 \pm 0.56$ & 2.95 & 4.13 & 2.00 & 44.9 & -0.08 \\
\hline G332.77-00.01 & $16: 17: 31.13$ & $-50: 32: 35.7$ & $5.67 \pm 0.57$ & 3.22 & 4.16 & 1.14 & 24.6 & $\ldots$ \\
\hline G337.63-00.08 & $16: 38: 19.02$ & $-47: 04: 51.0$ & $3.82 \pm 0.43$ & 2.88 & 4.01 & 2.20 & 31 & 0.01 \\
\hline G337.84-00.37 & $16: 40: 26.68$ & $-47: 07: 13.1$ & $2.98 \pm 0.47$ & 2.55 & 4.59 & 3.32 & 11.1 & 0.63 \\
\hline G336.98-00.18 & $16: 36: 12.43$ & $-47: 37: 58.0$ & $4.67 \pm 0.41$ & 2.51 & 4.43 & 1.47 & 18 & 0.52 \\
\hline G339.11+00.15 & $16: 42: 59.58$ & $-45: 49: 43.6$ & $4.96 \pm 0.40$ & 2.93 & 4.24 & 1.98 & 42.9 & -0.04 \\
\hline
\end{tabular}

Notes. The luminosity in Col. 7 is that of the HII region, and the final column gives the radio derived spectral index. For the targets which were not detected in all of the ATCA and VLA wavebands in Urquhart et al. $(2007,2009)$, no spectral index $(\alpha)$ was determined. ${ }^{(a)}$ Integrated Flux at $6 \mathrm{~cm}$.

Table 3. Spectral Setup of the observed regions.

\begin{tabular}{cccccc}
\hline \hline SPW & $\begin{array}{c}\text { Freq } \\
(\mathrm{GHz})\end{array}$ & Species & N. Chan & $\begin{array}{c}\text { Vel. BW } \\
\left(\mathrm{km} \mathrm{s}^{-1}\right)\end{array}$ & $\begin{array}{c}\text { Vel. Res. } \\
\left(\mathrm{km} \mathrm{s}^{-1}\right)\end{array}$ \\
\hline 1 & 257.403 & $\mathrm{CH}_{3} \mathrm{CN}$ & 3840 & 545.8 & 0.284 \\
2 & 257.127 & $\mathrm{CH}_{3} \mathrm{CN}$ & 1920 & 273.2 & 0.285 \\
3 & 256.302 & $\mathrm{H} 29 \alpha$ & 1920 & 274.1 & 0.258 \\
4 & 244.936 & $\mathrm{CS}$ & 1920 & 286.8 & 0.299 \\
5 & 242.998 & $\mathrm{SO}_{2}$ & 1920 & 289.1 & 0.301 \\
6 & 241.616 & $\mathrm{SO}_{2}$ & 960 & 145.4 & 0.303 \\
\hline
\end{tabular}

Notes. The primary targetted species of each spectral window are listed, with the note that spectral windows 1 and 2 were combined to get the full $\mathrm{CH}_{3} \mathrm{CN}(\mathrm{J}=12-11) \mathrm{k}$-ladder.

In Table 2 we present the radio parameters of the Hil regions and their host cores. The observing pointing centres are taken from the position of the peak radio flux densities and the distances with their associated uncertainties are computed using the Reid et al. (2016) rotation model. The masses and bolometric luminosities are recalculated from the values given in Urquhart et al. (2014) using the updated source distance and assuming an isothermal dust temperature of $30 \mathrm{~K}$ on single dish scales. Allowing for a variation in the temperature of $\pm 5 \mathrm{~K}$ and taking account of distance uncertainties, we estimate a measurement uncertainty in the mass of $\sim 30-50$ percent, while other systematics could be pushing the formal uncertainties to a factor of $2-3$.

The ALMA data presented here (from project 2013.1.00327.S, PI: P. Klaassen) consists of two groupings of targets based on co-observability on the sky (groups of HII regions within $10^{\circ}$ of each other). Each group was observed with the atacama compact array (ACA) and in two configurations with the $12 \mathrm{~m}$ baseline array (one compact, one extended) covering baseline lengths from $15 \mathrm{~m}$ to $\sim 784 \mathrm{~m}$. The integration times, calibrators (and their purposes), observing date, and minimum and maximum baseline lengths used in each of the executions contained in this set of observations are listed in Table A.1. The spectral set-up is given in Table 3. G339.11+00.15 was not observed in the most extended configuration. Hereafter, when referring to the targets of this study, we refer to such target with a shortened name consisting of the targets Galactic longitude (e.g. G302.02+00.25 becomes G302.02).
To ensure proper weighting of the different configurations when combining datasets, all of the data were manually reduced (i.e. not using the ALMA pipeline) in CASA 4.2.2 (McMullin et al. 2007). For each execution block, calibrations were made for water vapour, system temperatures, and antenna positions followed by bandpass, phase, and amplitude (flux) calibration. In the instances of multiple executions (e.g. ACA observations) an additional level of flux calibration was conducted to ensure consistent fluxes across each execution.

For each target and in each configuration, the continuum emission was strong enough for self-calibration, which was done in phase only. Additional amplitude self-calibration was found to not improve the signal-to-noise ratio, and was therefore omitted. The only exception was G332.77, for which self-calibration was not possible (peak $S / N=13$ ) in the highest resolution dataset.

The continuum emission was derived from the line free portions of each spectral window and then split out for each science target (using uvcontsub), and used for self-calibration of the data. These self-calibration solutions were then applied to the line data.

\section{Results}

We present analyses of some of the data observed with ALMA in this paper. A full analysis of all the data obtained for this project and their implications are presented in follow-up papers. In this work, we focus on the continuum emission, ionised gas observed within the HII regions, and some of the small scale molecular emission (in $\mathrm{CS}$ and $\mathrm{CH}_{3} \mathrm{CCH}$ ) surrounding the HiI regions to put the analysis of the ionised gas into context. Full analysis of the molecular environments and the chemistry of these regions is left to future work (e.g. Klaassen et al., in prep.; van der Tak et al., in prep.).

The analysis is broken down into a number of subsections; continuum (Sect. 3.1), ionised and molecular gas components (Sects. 3.2, 3.3 and 3.4) are discussed here. Because the ionised and molecular spectra (primarily the $\mathrm{H} 29 \alpha$ and $\mathrm{CH}_{3} \mathrm{CCH}$ ) were blended, there is an additional section detailing how their emission components were disentangled that is presented in Appendix B.

Figures 1 through 9 show the $256 \mathrm{GHz}$ continuum emission and bulk molecular gas emission (as traced by CS) for each of the regions studied here. Additionally, for those sources for which H29 $\alpha$ was detected (Figs. 1-6), we show both maps and spectra of this emission. 


\subsection{Continuum and cores}

\subsubsection{Core images}

In the top left panels of Figs. 1 through 9, we present the $256 \mathrm{GHz}$ continuum emission for each of our observed $\mathrm{HII}$ regions. Overplotted in red contours is the $5 \mathrm{GHz}$ emission (see Sect. 3.1.3) and the scale bar in the top left corner represents the thermal Jeans Length in each region (see Sect. 3.1.4). The cores identified in each region are shown with yellow contours and the number of each core is labelled in white from brightest to dimmest. These are numbered in the same order as presented in Table A.2, which presents the derived core properties in each region.

\subsubsection{Radio continuum and SED analysis}

The radio emission is relatively compact, is typically of a few arc-seconds in size, and has fluxes of up to $\sim 60 \mathrm{mJy}$ at $5 \mathrm{GHz}$ (see Table A.2). All but two of the targets have both 5 and 8.6 $\mathrm{GHz}$ detections with ATCA. For those detected at both frequencies, spectral indices $(\alpha)$ were calculated and generally found to be of order zero, as expected for optically thin thermal emission. There are two exceptions (G336.98 and G337.84), whose spectral indices are closer to one (see Table 2), and are indicative of optically thin thermal emission (e.g. Zapata et al. 2008) or ionised jets (e.g. Purser et al. 2016). In the analysis presented here, we favour the optically thin scenario over the ionised jet because of the relationship between the $8 \mathrm{GHz}$ and IR luminosities of these targets. When plotted against each other (as in Fig. 6 of Hoare \& Franco 2007), these targets, with $L_{8 \mathrm{GHz}}>10^{13} \mathrm{~W} \mathrm{~Hz}^{-1}$ and $L_{b o l}>10^{4} L_{\odot}$, lie in the HC HII region regime, more than one dex in $L_{8 G H z}$ above the jet sources. These are likely the youngest HII regions in our sample and are also the regions directly associated with the masers plotted in Figs. 1-5.

Using the radio spectral indices, we quantified the amount of free-free emission expected in our $256 \mathrm{GHz}$ continuum observations. Subtracting that value from the observed emission results in the thermal dust component of the emission from each region (see Table 5). In most cases, the free-free component represents more than $50 \%$ of the $256 \mathrm{GHz}$ continuum flux for the mm core associated with the HII region (see the core analysis below in Sect. 3.1.3).

\subsubsection{Core boundaries and masses}

Each of the systems observed in this sample appears to have at least moderately $(>2)$ clumpy structures in the continuum. In quantifying that so-called clumpiness, we can determine properties about the cluster, including its structure and the mass of each core. To do this, we used the Fellwalker package (Berry 2015), which uses a watershed algorithm to determine the edges of the boundaries between cores in the input continuum image. This watershed algorithm finds the steepest downwards gradients in emission from each peak (like water running down a hill) to find where it pools (at the noise threshold). The final multi-configuration continuum images were clipped at $3 \sigma$ (with $\sigma$ given in Table 1), and in each case, the algorithm found at least two cores in each image. The cores themselves are shown with yellow contours and are numbered in white in the top left panels of Figs. 1 to 9. The properties of the cores associated with the HII regions are shown in Table 5 and the full listing of core properties is presented in Table A.2.

The outputs of Fellwalker include the positions of the peaks of each core, the enclosed area (in square arcsec), and summed intensity within the core. The resultant intensities are listed in Table A.2. From the flux of each core, and certain assumptions about the targets (such as filled beams and ambient temperatures), we derived core masses as described below.

The mass of each core is derived under the assumption that the emission is dominated by the thermal dust continuum, using Eq. (6) of Hildebrand (1983) such that

$M_{c}=\left(\frac{F_{v} D^{2}}{B(v, T)}\right)\left(\frac{4}{3} \frac{a}{Q v}\right) \rho\left(\frac{M_{g}}{M_{d}}\right)$,

where $F_{v}$ is the observed dust flux density, $D$ is the distance to source, $B(v, T)$ is the Planck blackbody dust intensity, $a$ is the assumed dust grain size, $Q_{v}$ is the dust emissivity, and $M_{g} / M_{d}$ is the gas to dust mass ratio (assumed to be 100; e.g. Draine 2004).

In Table A. 2 we show the results of these calculations at three temperatures $(20,50$, and $100 \mathrm{~K})$ to show how the masses change with assumed temperature. There is only potential for free-free emission contamination in the mass estimates for (at most) one core in each cluster. The free-free corrected values are presented in Table 5 for the contaminated cores.

We additionally use Eq. (1) to quantify our point source mass sensitivity in a single beam with the rms noise limits listed in Table 1 to find that we are sensitive to masses and column densities at the $10^{-2} M_{\odot}$, or $3.6 \times 10^{21} \mathrm{~cm}^{-2}$ limits, respectively.

\subsubsection{Core separation and Jeans lengths}

The clumpy nature of the continuum emission allowed us to analyse the fragmentation of each region on scales of the Jeans lengths (see e.g. Wang et al. 2014). While we did not have enough cores to create a significant core mass function, we can compare the observed fragmentation scales to the Jeans length in each region.

The Jeans lengths were calculated using

$$
\begin{aligned}
& \lambda_{J}=\left(\frac{\pi c_{s}^{2}}{G \rho}\right)^{1 / 2} \\
& \lambda_{J}=0.4 \mathrm{pc} *\left(\frac{c_{s}}{0.2 \mathrm{~km} \mathrm{~s}^{-2}}\right)\left(\frac{10^{3} \mathrm{~cm}^{3}}{n}\right)^{1 / 2},
\end{aligned}
$$

where $c_{s}$ is the sound speed, $\rho$ is the average mass density in each region, and $n$ is the volume number density of each region. The sound speed can be calculated from the temperature of each region as $c_{s}=\sqrt{(k T / m)}$, where $k$ is the Boltzmann constant and $m$ is the mean molecular mass of the gas (set here to $2.8^{*} m_{H}$, Kauffmann et al. 2008).

The sound speed and volume density of the molecular gas were calculated from the XCLASS analysis of the $\mathrm{CH}_{3} \mathrm{CCH}$ presented in Sect. B.1, and roughly correspond to $0.5 \mathrm{~km} \mathrm{~s}^{-1}(T \sim$ $70-100 \mathrm{~K}$ ) and $\sim 10^{7} \mathrm{~cm}^{-3}$ (from the column density and the assumption of spherical symmetry). This gives thermal Jeans lengths of $\sim 2000$ au in each region, which is a lower limit because the volume density of the progenitor cores was lower. The larger scale densities, from which these cores fragmented, are much closer to $10^{5} \mathrm{~cm}^{-3}$ (see, for instance, Csengeri et al. 2016), resulting in a Jeans length 10 times larger, and more consistent with the fragmentation lengths seen in these regions. These values are presented in Table 4. The comparison can also be made to the turbulent Jeans length (see e.g. Wang et al. 2014). In this instance, the turbulent line width (standard deviation of the line width, not the FWHM) replaces the thermal line width 
P. D. Klaassen et al.: The evolution of young Hil regions. I.

Table 4. Derived Jeans lengths (given in au), and core separation statistics, including the mean and standard deviation $(\sigma)$ of the core separations.

\begin{tabular}{cccccccc}
\hline \hline Target & No. clumps & $L_{\text {Jeans }}$ & Mean Sep. & $\sigma$ & Min Sep. & $L_{\text {Jeans }} /$ Mean Sep. & Max Sep. \\
\hline G302.02 & 13 & 18740 & 17900 & 6500 & 5000 & 1.05 & 27400 \\
G302.48 & 11 & 18740 & 12900 & 6800 & 5700 & 1.45 & 28200 \\
G309.89 & 12 & 18740 & 22600 & 9900 & 12500 & 0.83 & 40300 \\
G330.28 & 7 & 20772 & 27400 & 13800 & 10800 & 0.76 & 50900 \\
G332.77 & 2 & 18740 & 38700 & 0 & 38700 & 0.48 & 38700 \\
G336.98 & 4 & 12867 & 11200 & 5300 & 7000 & 1.15 & 18600 \\
G337.63 & 7 & 22286 & 9100 & 5000 & 3000 & 2.45 & 17600 \\
G337.84 & 9 & 18740 & 13400 & 7500 & 4600 & 1.40 & 25400 \\
G339.11 & 5 & 15838 & 27200 & 10900 & 15100 & 0.58 & 40100 \\
\hline
\end{tabular}

Notes. Generally, the Jeans length $\left(L_{\text {Jeans }}\right)$ is resolved in these observations. The Turbulent Jeans length, for comparison, is of order 60000.0 AU.

Table 5. Radio (5 GHz) flux of each HII region and spectral $\alpha$ (updated from Urquhart et al. (2014)).

\begin{tabular}{lrrrrrr}
\hline \hline Source & $S_{5 \mathrm{GHz}}$ & $\alpha$ & $S_{\text {tot }}$ & $S_{\text {FF }}$ & $S_{\text {dust }}$ & $M_{\text {dust }}$ \\
\hline G302.02 & 59.5 & -0.03 & 62.1 & 53.2 & 8.9 & 29.6 \\
G302.48 & 23.4 & 0.03 & 93.9 & 26.2 & 67.7 & 142.4 \\
G309.89 & 1.2 & - & 36.3 & - & - & - \\
G330.28 & 44.9 & -0.08 & 79.9 & 33.3 & 46.6 & 253.5 \\
G332.77 & 24.6 & - & 3.5 & - & - & - \\
G336.98 & 18.0 & 0.52 & 243.9 & 126.7 & 117.1 & 312.8 \\
G337.63 & 31.0 & 0.01 & 53.1 & 32.2 & 20.9 & 33.9 \\
G337.84 & 11.1 & 0.63 & 135.3 & 118.1 & 17.2 & 68.5 \\
G339.11 & 42.9 & -0.04 & 189.3 & 36.9 & 152.4 & 686.0 \\
\hline
\end{tabular}

Notes. The total flux from the HII region bearing Fellwalker core $\left(S_{\text {tot }}\right)$ is subsequently listed as a reminder from Table A.2 because the free-free extrapolated flux $\left(S_{\mathrm{FF}}\right)$ is then removed from the $256 \mathrm{GHz}$ continuum flux to give the expected contribution to the continuum emission $\left(S_{\text {dust }}\right)$. All fluxes are listed in units of mJy. Using the dust mass assumptions described in Sect. 3.1.3 and a temperature of $70 \mathrm{~K}$, the resultant dust masses are presented in units of $M_{\odot}$.

(sound speed) in Eq. (3). This turbulent Jeans length corresponds to $\sim 0.3$ pc using large scale densities.

With the results of the Fellwalker analysis presented above, we can quantify the separation between the cores using a minimum spanning tree method (e.g. Allison et al. 2009). Using the core peak coordinates listed in Table A.2, we determined the spacing between cores using the algorithms available in astroML (VanderPlas et al. 2012). The mean (and standard deviation, $\sigma$ ) of these separations are given in Table 4, and these separations can then be compared to the Jeans length for each region, as given in Table 4.

In all cases except G339.11, for which we have lower resolution data, we resolved the thermal Jeans length. Of the eight regions with high enough resolution observations, all have mean (and indeed minimum) core separations greater than the thermal Jeans length. However, half of the regions have minimum core separations similar to the turbulent Jeans length (see Table 4), which suggests that turbulence was a dominant factor in determining the fragmentation in these regions.

\subsection{Ionised gas}

Often pressure broadening dominates the line widths of radio recombination lines (RRLs) at longer wavelengths. This pressure broadening comes primarily from the electrons in dense HII regions (cf. Brocklehurst \& Seaton 1972; Brown et al. 1978), which for a $n_{e}=10^{6} \mathrm{~cm}^{-3}$ HII region, can result in line widths greater than $400 \mathrm{~km} \mathrm{~s}^{-1}$ (for $\mathrm{Hn} \alpha$, where $n=100$, at $\sim 6.5 \mathrm{GHz}$ ). However, in our case (with $n=29$ ), the pressure broadening contribution for such an HII region is only a few $\mathrm{m} \mathrm{s}^{-1}$. Pressure broadening, which scales linearly with electron density, manifests as a Lorentzian line profile, and not a Gaussian line profile as many other broadening mechanisms do (see e.g. Keto et al. 2008; Galván-Madrid et al. 2012). The result of the blend of Gaussian and Lorentzian profile is a Voigt profile. For our $\mathrm{H} 29 \alpha$ observations we performed both Gaussian and Voigt profile fitting and find our data are best fit by Gaussian profiles alone (or Voigt profiles with no Lorentzian component). From these pressure broadening non-detections, we can place constraints on a number of HII region properties, including temperature and electron density. For these constraints, we use the larger of the uncertainty on the Gaussian width of the $\mathrm{H} 29 \alpha$ line and the velocity resolution of our observations $\left(0.25 \mathrm{~km} \mathrm{~s}^{-1}\right)$.

The $\mathrm{H} 29 \alpha$ spectra (averaged over the emission area), best fit Gaussian profiles, and resulting residuals are all presented in the bottom left panels of Figs. 1 to 6, with the Gaussian parameters presented in Table 6, where the expected molecular $V_{\mathrm{LSR}}$ is also given for reference.

It appears that for each region with an $\mathrm{H} 29 \alpha$ detection, the RRL emission is offset from the reported source $V_{\mathrm{LSR}}$ (e.g. those given in Table 6) by a few $\mathrm{km} \mathrm{s}^{-1}$, suggesting either that the source velocity is uncertain by a few $\mathrm{km} \mathrm{s}^{-1}$, or that the HII region is moving at a slightly different velocity to its surroundings. It is likely that there is a mixture of these two cases, as the CS emission shown for the same areas in Figs. 1-9 are a mixture of having the same offsets (e.g. G302.02), which are centred at the same velocities but with smaller velocity ranges (e.g. G337.63) and are consistent with the LSR velocities previously known for the source and offset from the RRL emission (e.g. G337.84).

Below we describe the detection rate of $\mathrm{H} 29 \alpha$ (Sect. 3.2.1), properties of the HII regions we derived from the $\mathrm{H} 29 \alpha$ emission (Sect. 3.2.2), and our analysis of the velocity gradients seen in each region (Sect. 3.2.3), and their implications.

\subsection{1. $R R L$ detection rate}

After the fitting and removal of molecular contamination, $\mathrm{H} 29 \alpha$ was detected in a total of six out of nine of our HII regions. The top right panels of Figs. 1 through 6 show the integrated intensity (moment zero) and intensity weighted velocity (moment 
one) maps of the $\mathrm{H} 29 \alpha$ emission in each of the six regions, where regions that have $\mathrm{CH}_{3} \mathrm{CCH}$ subtraction applied are noted with asterisks. In all but one case (G337.63), the H29 $\alpha$ emission is at or near the position of the brightest continuum source. For G337.63, the $\mathrm{H} 29 \alpha$ emission is detected towards the fourth brightest continuum peak (labelled as "4" in Fig. 4). We note that for G330.28, the $\mathrm{H} 29 \alpha$ is slightly offset from the continuum peak, however, it is still localised to the brightest core within the observed field of view.

There are a number of reasons why the $\mathrm{H} 29 \alpha$ was not detected in three of our targets. For G332.77, our signal-to-noise ratios are generally (e.g. in the continuum and molecular emission) very low, suggesting any RRL emission would be below our detection threshold. This is not the case for G302.48 and G309.89, for which the continuum and molecular emission are well detected. This then suggests that the nature of these sources is what leads to the non-detections. The principle physical reasons are related to potentially low temperatures, HII regions that are too diffuse (i.e. old) for the detection of the high density lines, or that the star(s) powering the HII regions are not (yet) massive enough to produce high frequency RRLs. G309.89 for instance, has been detected in $\mathrm{H} 109 \alpha$ and $\mathrm{H} 110 \alpha$ (Caswell \& Haynes 1987), but remains undetected in $\mathrm{H} 29 \alpha$ in our sample. Using the first equation in Sect. 3 of Caswell \& Haynes (1987), their derived temperature of $6400 \mathrm{~K}$ for this region, and our $256 \mathrm{GHz}$ continuum peak for the core (36 mJy, see Table A.2) as an upper limit to the unknown fraction of the continuum that comes from free-free emission, we find an expected $\mathrm{H} 29 \alpha$ line peak below our line sensitivity threshold.

\subsubsection{Derived HII region properties}

If we assume that the Gaussian line width is purely thermal, we can derive an upper limit to the electron temperature required to produce the observed line width. These values are presented in Table 7. For $n=29$, the natural line width is of order $1 \mathrm{~m} \mathrm{~s}^{-1}$ and therefore is not a significant contribution to the line width (see e.g. Chapter 2 of Gordon \& Sorochenko 2002).

With an upper limit to the pressure broadening coming from the uncertainties on the Gaussian fits to the H29 $\alpha$ emission, an upper limit on the electron density is derived via (Brocklehurst \& Seaton 1972)

$\frac{\Delta v_{I}}{v_{0}}=1.43 \times 10^{-5}\left(\frac{n}{100}\right)^{7.4}\left(\frac{10^{4} \mathrm{~K}}{T}\right)^{0.1}\left(\frac{n_{e}}{10^{4} \mathrm{~cm}^{3}}\right)$,

where $\Delta v_{I}$ is the upper limit to the pressure broadened width of the line, $n$ is the quantum number of the transition $(n=29), T$ is the electron temperature (in $\mathrm{K}$ ) derived above, and $n_{e}$ is the electron density (in $\mathrm{cm}^{-3}$ ). Through this equation, we see that the lack of pressure broadening constrains the electron densities in these regions to be less than $5 \times 10^{7} \mathrm{~cm}^{-3}$. The electron density requirement for a region to be classed as hyper compact is of order $10^{6} \mathrm{~cm}^{-3}$ (Hoare et al. 2007; Kurtz 2005), which is lower than the limits suggested by our observations, and it is therefore conceivable that these are indeed still hyper-compact HII regions. This is consistent with the sizes of the $\mathrm{H} 29 \alpha$ emitting regions as they are all smaller than the 10000 au approximate upper limit on the size of an HCHII region (see for instance Hoare et al. 2007).

With the electron density upper limit in hand, we can further (loosely) extrapolate to upper limits on the mass of the gas in each HII region (but not the mass of the ionising source). From the area enclosed in the moment zero maps presented in
Figs. 1-6 and the assumption of spherical symmetry, we can approximate a volume for each HII region. Multiplying that volume by the electron density (which should be the same as the proton density in the HII region), we determine the number of free protons in the HII region. This can then be translated to the HII region masses presented in Table 7 . We find that these limits are very high, which suggests that the upper limits on the electron densities (especially in the case of G339.11) are not very strict (i.e. that the electron densities could be an order of magnitude lower).

An estimate of the free-free continuum emission can be quantified from the integrated line flux and temperature (Brown et al. 1978) as follows:

$\frac{\int T_{L} \mathrm{dv}}{T_{c}} \sim 6.76 \times 10^{3} v^{-1.1} T_{\mathrm{e}}^{-1.15}$,

where the integrated line flux $\left(\int T_{L} \mathrm{dv}\right)$ is taken from the Gaussian fits presented in Table 6 and is transformed into units of mJy using the synthesised beam sizes presented in Table 1 .

The free-free continuum emission levels derived using this method are anywhere between five and 40 times lower than those derived from extrapolating the radio spectral index to the $\mathrm{mm}$. It should be noted that this equation assumes optically thin emission, which, as we see in Sect. 3.3, may not be the case for at least some of our HiI regions.

\subsubsection{RRL velocity gradients}

As can be seen in the first moment maps of $\mathrm{H} 29 \alpha$ (Figs. 1 through 6), none of our HII regions have the linear velocity gradients usually associated with rotation. The resolution achieved for G339.11 is much coarser than that for the others (of order $1.4^{\prime \prime}$ instead of $\sim 0.5^{\prime \prime}$ ) so its dynamical/morphological type is less clearly defined. Its appearance (see Fig. 6) does not suggest it to be cometary, and whether it has the same dynamical signature of infall on the large size scales probed by our observations is unclear. Higher resolution observations would be required to distinguish this from multiple cores with our beam.

Our $\mathrm{H} 29 \alpha$ detection in G337.84 is a weak detection and therefore we cannot properly constrain a velocity gradient. For G330.28 and G337.63, the first moment maps of the RRL emission are more indicative of a constant velocity bow/arc with a blue or red (respectively) tail. In these cases, indeed, the integrated intensity maps of the RRL emission are also the most cometary in appearance. These velocity structures are similar to those seen by Immer et al. (2014) in H66 $\alpha$ in DR21 on much larger scales (in a much larger and older HII region), which they interpret as being due to bow shocks.

It is also in these regions that we see the CS emission wrapping around the HII region (see Figs. 2 and 4), with a dearth of emission 'downwind' from the HII region. In general, the peak of the CS emission is offset from the peak of the H29 $\alpha$ emission. However it is in these two regions (G330.28 and G337.63) that the most striking offsets occur: the CS appears to be upwind (i.e. closer to the bow than the tail) of the HII region and little to no emission overlaps with the HII region itself. If the HII region is pushing through the ambient material, this offset is likely due to the molecular gas having been compressed at the head of the cometary region, which leaves a cavity in its wake. If the offset is caused by expansion of the HII region, then it is a product of the density gradient, where one edge of the region pushes against (and compressing) the dense material while the other edge is 
Table 6. Gaussian fits to $\mathrm{H} 29 \alpha$ emission.

\begin{tabular}{rccrr}
\hline \hline Target & $\begin{array}{c}\text { Amp. } \\
(\mathrm{mJy})\end{array}$ & $\begin{array}{c}\text { Central velo. } \\
\left(\mathrm{km} \mathrm{s}^{-1}\right)\end{array}$ & $\begin{array}{r}V_{\mathrm{LSR}} \\
\left(\mathrm{km} \mathrm{s}^{-1}\right)\end{array}$ & $\begin{array}{r}\text { Line width } \\
\left(\mathrm{km} \mathrm{s}^{-1}\right)\end{array}$ \\
\hline G302.02 & $9.7 \pm 0.1$ & $-46.0 \pm 0.2$ & -37.1 & $11.4 \pm 0.2$ \\
G330.28 & $1.2 \pm 0.2$ & $-99.1 \pm 1.8$ & -93.5 & $12.3 \pm 1.8$ \\
G336.98 & $3.2 \pm 0.2$ & $-95.1 \pm 0.0$ & -75.1 & $13.6 \pm 0.0$ \\
G337.63 & $3.6 \pm 0.2$ & $-53.5 \pm 0.5$ & -56.5 & $9.3 \pm 0.5$ \\
G337.84 & $6.6 \pm 1.0$ & $-42.2 \pm 1.4$ & -40.4 & $8.2 \pm 1.4$ \\
G339.11 & $5.1 \pm 0.4$ & $-90.4 \pm 1.7$ & -78.2 & $13.6 \pm 0.0$ \\
\hline
\end{tabular}

Table 7. Derived HII region properties based on H29 $\alpha$ emission.

\begin{tabular}{lrcrrr}
\hline \hline Target & $\begin{array}{r}T \\
(\mathrm{~K})\end{array}$ & $\begin{array}{r}\Delta v_{p} \\
\left(\mathrm{~km} \mathrm{~s}^{-1}\right)\end{array}$ & $\begin{array}{r}n_{e} \\
\left(10^{7} \mathrm{~cm}^{-3}\right)\end{array}$ & $\begin{array}{r}M_{\mathrm{HII}} \\
M_{\odot}\end{array}$ & $\begin{array}{r}S_{c} \\
(\mathrm{mJy})\end{array}$ \\
\hline G302.02 & 2800 & 0.25 & $<1.5$ & $<38.4$ & 11 \\
G330.28 & 3300 & 1.8 & $<11$ & $<482.9$ & 2.3 \\
G336.98 & 4100 & 0.25 & $<1.5$ & $<274.0$ & 8.7 \\
G337.63 & 1900 & 0.54 & $<3.0$ & $<80.2$ & 2.7 \\
G337.84 & 1500 & 1.4 & $<7.7$ & $<220.1$ & 3.4 \\
G339.11 & 4100 & 0.25 & $<1.5$ & $<1206.7$ & 1.9 \\
\hline
\end{tabular}

Notes. Temperatures derived assuming line widths are thermal, and voigt profile widths are taken to be the greater of half the channel width $\left(0.25 \mathrm{~km} \mathrm{~s}^{-1}\right)$ or the uncertainty on the Gaussian fit in Table 6 .

able to break out of the ambient gas and push out the less dense material as it expands.

For two of our targets, (G302.02 and G336.98, and potentially G339.11, but at lower resolution), the RRL shows a concentric velocity gradient, the so-called "bulls-eye" morphology (see, for instance, Sollins et al. 2005). Similar (e.g. redshifted towards the centre) profiles were predicted for optically thick RRL emission in infalling HII regions by Peters et al. (2012, see Sect. 3.3), suggesting these velocity gradients are consistent with infalling ionised gas.

For these regions, we suggest that the bulls-eye features are infalling and not contracting because, according to De Pree et al. (2014), a contracting or collapsing HII region should be distinguished by a decrease in flux over time, not as an inwards velocity gradient or change in size of the HII region. These regions should be monitored over the coming decades to properly distinguish between these cases.

From the moment one maps of these two regions (G302.02 and G336.98 in Figs. 1 and 3, respectively), the velocity shifts from the edges to the centres of these HII regions range from $\sim 7$ to $10 \mathrm{~km} \mathrm{~s}^{-1}$, respectively. These are large on the $3000-4000 \mathrm{au}$ scales of these HII regions and correspond to velocity gradients of $300-500 \mathrm{~km} \mathrm{~s}^{-1} \mathrm{pc}^{-1}$.

The most surprising result of this study is the high percentage (up to $30 \%$, if the gradient in G339.11 is to be believed) of HII regions detected in $\mathrm{H} 29 \alpha$ that show infall signatures, when it was expected (from statistical lifetime arguments: Mottram et al. 2011a; Davies et al. 2011) that the stars powering these HII regions would have rather short (few $\times 10^{4} \mathrm{yr}$ ) ionised accretion lifetimes. However, though the Kelvin Helmholtz contraction timescale for single non-accreting sources of these luminosities $\left(\log (\mathrm{L})\right.$ of 4.2 and 4.4 , corresponding to $\sim 14$ and $\left.17 M_{\odot}\right)$ is of the order $10^{5} \mathrm{yrs}$, if their mass was assembled more slowly with more modest accretion rates $\left(\sim 10^{-4} M_{\odot} \mathrm{yr}^{-1}\right)$ then the models predict that the swelling is more modest and such (proto)stars would reach a phase of main-sequence phase already at these sorts of masses (see e.g. Hosokawa et al. 2010). The chemical diversity of these two confirmed (and one tentative) infalling regions is also curious. Two are the most chemically rich in our study (G339.11, and G336.98, which, from its radio SED is expected to be one of our youngest HII regions), while the other (G302.02) shows no molecular emission across the HII region (neither in the $\mathrm{H} 29 \alpha$ nor CS passbands), suggesting that the complex molecules have been destroyed by the ionising radiation.

\subsection{Models of ionised gas}

Peters et al. (2012) implemented the modelling of RRL line profiles detectable at JVLA and ALMA wavelengths into RADMC-3D using non-LTE methods to understand line shapes. The test cases they used to demonstrate the code focussed on infalling and outflowing RRL emission, and under which conditions these signatures would be detectable.

These conditions require the observations of the optically thick RRLs to be of high resolution and sensitivity, and for the intrinsic line widths to be narrow enough for the asymmetries to be spectrally resolved (e.g. pressure broadening does not dominate the spectrum). In Fig. 14 of Peters et al., these authors outlined the parameter space in which line profile variations as a function of position can be attributed to dynamics, and find a parameter space in the top right of their figure where RRL emission is optically thick and the medium thermally supported, thereby requiring high frequency observations of the densest HII regions.

Our observations $\left(v \sim 256 \mathrm{GHz}, n_{e}<5 \times 10^{7} \mathrm{~cm}^{-3}\right)$ fall into this parameter space, and indeed, we do find these line asymmetries in some of our regions.

We reran their models for infalling gas as traced by $\mathrm{H} 29 \alpha$. These model results are shown in Fig. 10, where we present the zeroth and first moment maps along with normalised spectra taken at radial points along the optically thick to thin transition (outwards). The first moment map clearly shows red-shifted emission in the optically thick region. Because the emission is optically thick, we are probing the near side of the HII region and the fact that the emission is red-shifted indicates the gas is moving away from us; it is falling inwards onto the HII region. A qualitative comparisons to our data then suggests that the bullseye patterns we see in G302.02 and G336.98 are indicative of infall motions onto/through the HII region.

\subsection{Molecular gas dynamics}

\subsubsection{XCLASS fitting: Molecular results}

There were two molecular species fit during the XCLASS modelling (see Appendix B.1) that were parameterised well enough to constrain some of the properties of the warm molecular gas surrounding the HII regions. Towards G336.98, both $\mathrm{CH}_{3} \mathrm{CCH}$ (Fig. B.2) and $\mathrm{CH}_{3} \mathrm{OCHO}$ were well constrained with the best fit models giving temperatures of $\sim 70-80 \mathrm{~K}$ and column densities of $3-10 \times 10^{14} \mathrm{~cm}^{-2}$. The $\mathrm{CH}_{3} \mathrm{CCH}$ shows a velocity gradient across the area surrounding the HII region (roughly orientated E-W), which is different from the infall signature seen in the $\mathrm{H} 29 \alpha$. The $\mathrm{CH}_{3} \mathrm{OCHO}$ does not show a velocity gradient. There are two reasons why this could be the case; either there is no velocity gradient or, more likely the fit was not 

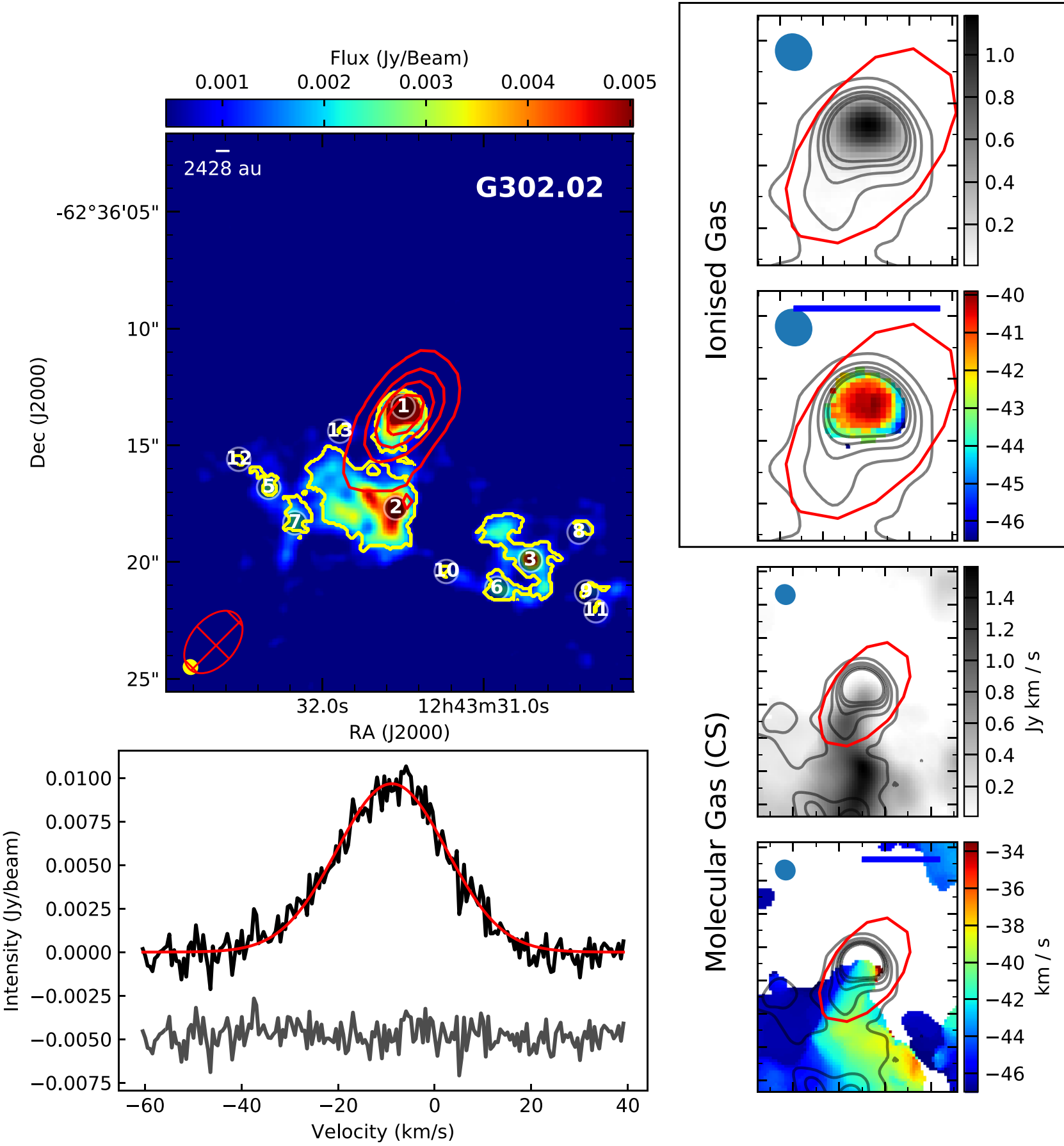

Fig. 1. Continuum, $\mathrm{H} 29 \alpha$, and CS emission from G302.02. Top left panel: $256 \mathrm{GHz}$ continuum in colour scale in which the $5 \mathrm{GHz}$ emission is shown in red contours; the red contours in the other images represent this same $5 \mathrm{GHz}$ emission for comparison. The yellow contours show the outlines of the FellWalker identified cores. Bottom left panel: H29 $\alpha$ emission (black) with its Gaussian fit. The grey line shows the residuals from the Gaussian fit. In these spectra, the emission has been recentred to the expected rest velocity of the source, thus the velocity shift of the line peak (from zero) represents a difference between the ionised and molecular gas velocities. Top two panels on the right: moment 0 and moment 1 maps of the $\mathrm{H} 29 \alpha$ emission (top and bottom), while bottom two panels on the right: moment 0 and moment 1 maps of the CS emission (top and bottom).

good enough to identify a velocity gradient since the $\chi^{2}$ values for $\mathrm{CH}_{3} \mathrm{OCHO}$ were much larger than for $\mathrm{CH}_{3} \mathrm{CCH}$. With $\mathrm{CH}_{3} \mathrm{CCH}$, there were five transitions available to constrain the velocity gradient and with $\mathrm{CH}_{3} \mathrm{OCHO}$, there is only one. Thus, it becomes easier for velocity, density, and temperature gradients to be mis-identified.
$\mathrm{CH}_{3} \mathrm{CCH}$ was also well constrained in $\mathrm{G} 302.48(T \sim 70 \mathrm{~K}$ $N \sim 10^{15} \mathrm{~cm}^{-2}$ ), but we can draw no comparison to the ionised gas dynamics as $\mathrm{H} 29 \alpha$ was not detected in this region.

$\mathrm{CH}_{3} \mathrm{OCHO}$ was detected in $\mathrm{G} 337.84$ (where a very faint detection of $\mathrm{H} 29 \alpha$ was also made) with suggestions of higher temperatures and column densities $\left(T \sim 300 \mathrm{~K}, N \sim 10^{15} \mathrm{~cm}^{-2}\right)$. 

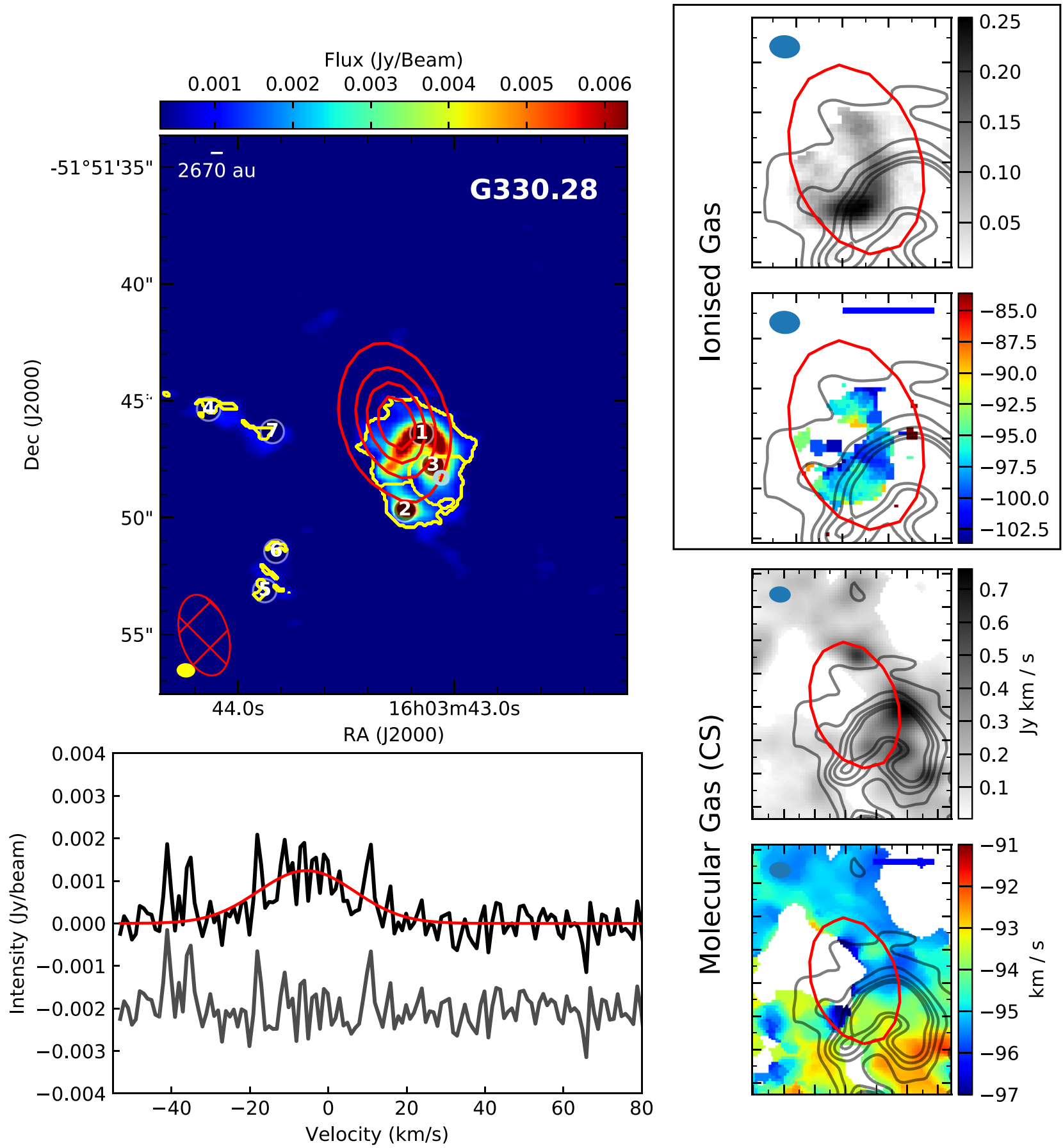

Fig. 2. Continuum, $\mathrm{H} 29 \alpha$, and CS emission from G330.28. Top left panel: $256 \mathrm{GHz}$ continuum in colour scale in which the 5 GHz emission is shown in red contours; the red contours in the other images represent this same $5 \mathrm{GHz}$ emission for comparison. The yellow contours show the outlines of the FellWalker identified cores. Bottom left panel: H29 $\alpha$ emission (black) with its Gaussian fit. The grey line shows the residuals from the Gaussian fit. In these spectra, the emission has been recentred to the expected rest velocity of the source, thus the velocity shift of the line peak (from zero) represents a difference between the ionised and molecular gas velocities. Top two panels on the right: moment 0 and moment 1 maps of the $\mathrm{H} 29 \alpha$ emission (top and bottom), while bottom two panels on the right: moment 0 and moment 1 maps of the CS emission (top and bottom).

Its velocity structure can be described as saddle-like and is reminiscent of the CS velocity patterns presented for this target below in Sect. 3.4.2, and in Fig. 5.

\subsubsection{Comparison to CS emission}

There were a number of molecular species observed along with $\mathrm{H} 29 \alpha$ in our observing set-up and the analysis of the larger scale molecular dynamics in these regions will be discussed in a forthcoming paper (Klaassen et al., in prep.). However, it is instructive to put the RRL emission into context with its surrounding molecular gas. In this light, we present a brief summary of the CS $(\mathrm{J}=5-4)$ dynamics surrounding each Hil region.

Integrated intensity and intensity weighted velocity (zeroth and first moment) maps are presented in Figs. 1-9 (right panels labelled as "molecular"). As can be seen from these maps, the 

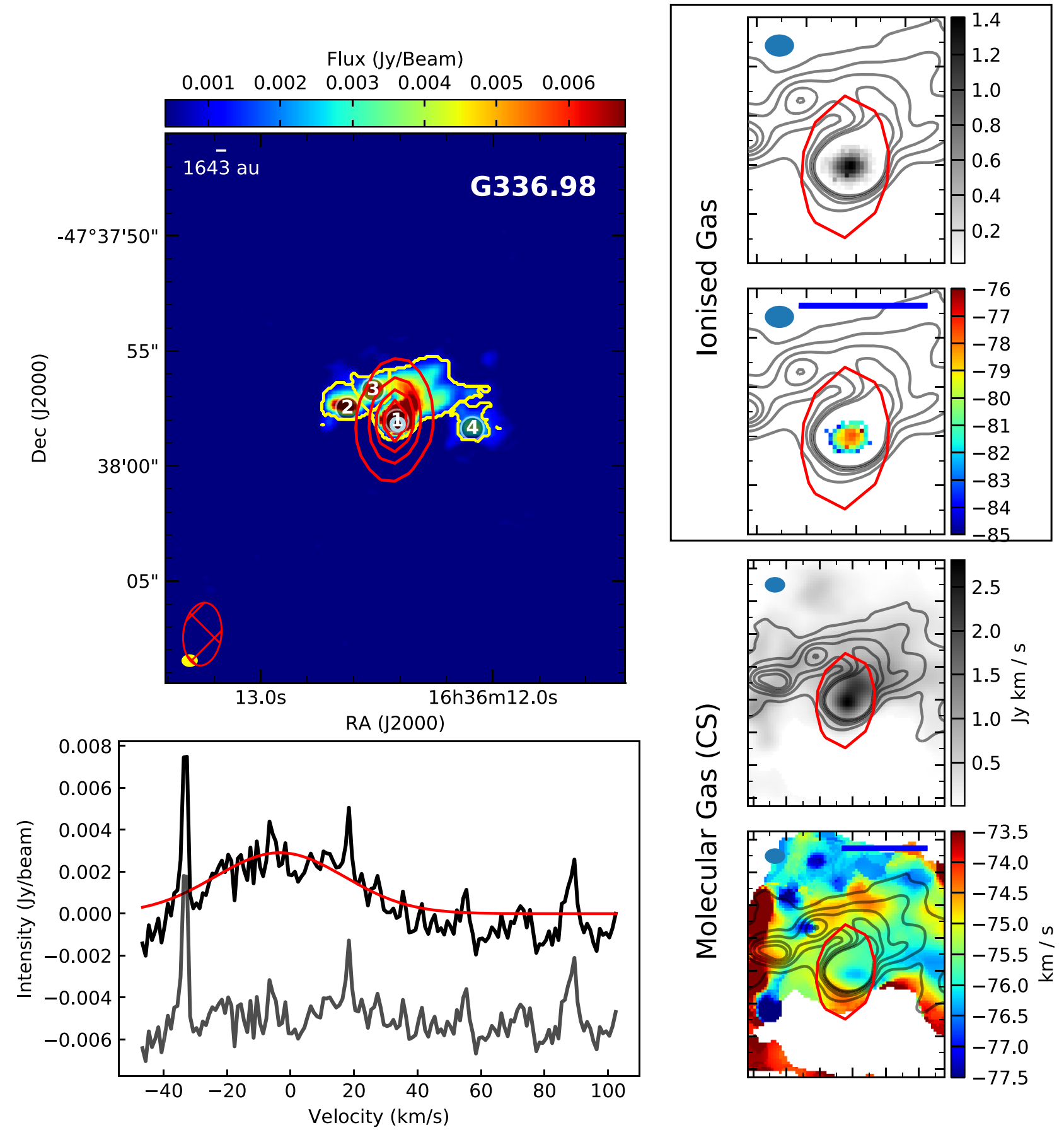

Fig. 3. Continuum, $\mathrm{H} 29 \alpha$, and CS emission from G336.98. Top left panel: $256 \mathrm{GHz}$ continuum in colour scale in which the $5 \mathrm{GHz}$ emission is shown in red contours; the red contours in the other images represent this same $5 \mathrm{GHz}$ emission for comparison. The yellow contours show the outlines of the FellWalker identified cores. Bottom left panel: $\mathrm{H} 29 \alpha$ emission (black) with its Gaussian fit. The grey line shows the residuals from the Gaussian fit. In these spectra, the emission has been recentred to the expected rest velocity of the source, thus the velocity shift of the line peak (from zero) represents a difference between the ionised and molecular gas velocities. Top two panels on the right: moment 0 and moment 1 maps of the $\mathrm{H} 29 \alpha$ emission (top and bottom), while bottom two panels on the right: moment 0 and moment 1 maps of the CS emission (top and bottom).

velocity ranges over which the CS emits are much smaller than those of the $\mathrm{H} 29 \alpha$.

For the HII regions with cometary morphologies, unsurprisingly the molecular emission also appears to have a somewhat cometary structure. In the two cases presented here, we note that the CS emission generally tends to be detected around the HII region, rather than coincident with it. This dearth of molecular emission suggests either that the ionised gas has cleared away a region for it to expand (i.e. a champagne flow) through the lower density medium, or that the HII region is moving through the molecular gas and creating a bow of molecular emission at its head. From the current data, we cannot break this degeneracy.

In the cases of the regions with suggested infalling motions in the ionised gas, the CS emission does not show much 

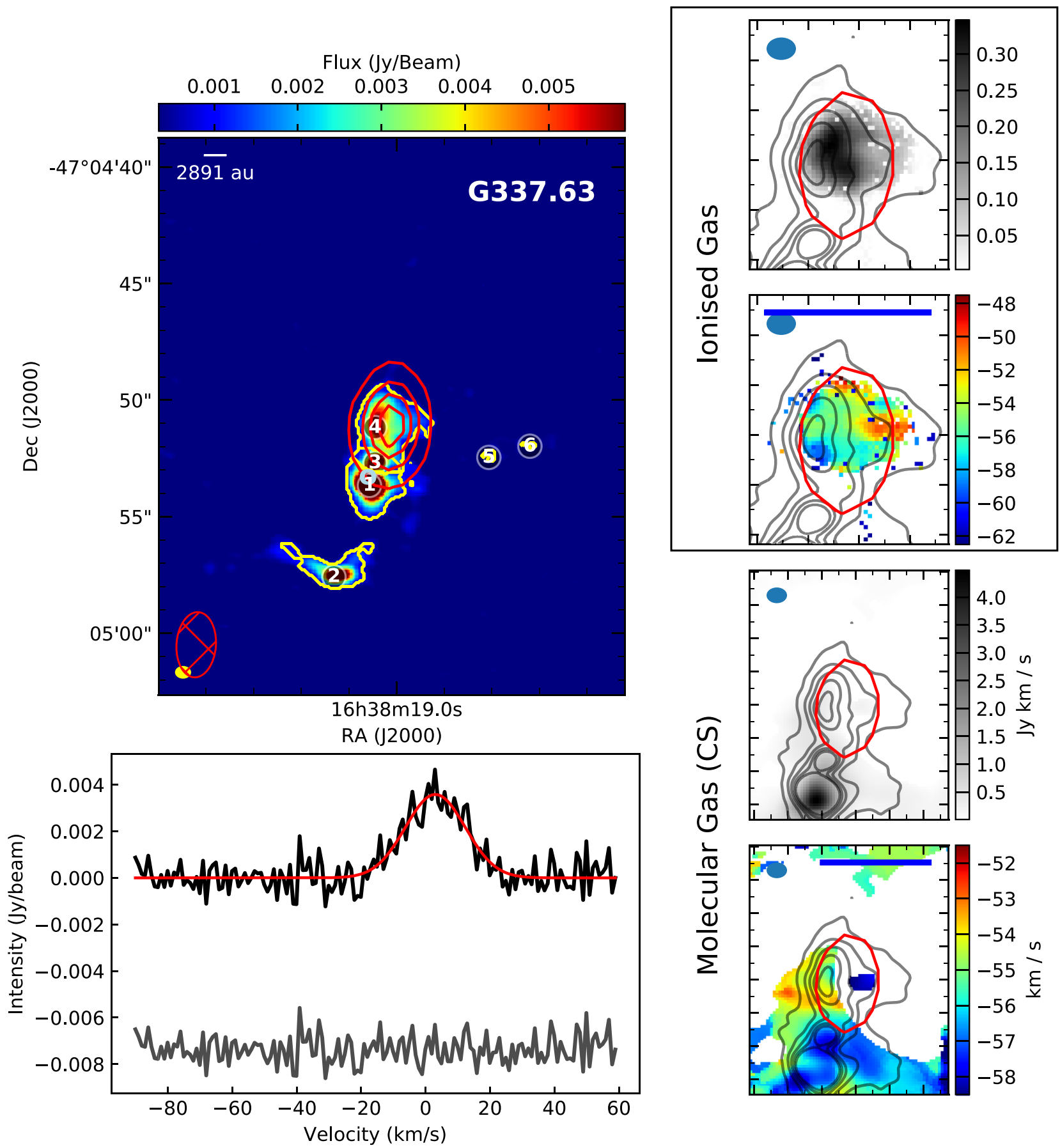

Fig. 4. Continuum, $\mathrm{H} 29 \alpha$, and CS emission from G337.63. Top left panel: $256 \mathrm{GHz}$ continuum in colour scale in which the $5 \mathrm{GHz}$ emission is shown in red contours; the red contours in the other images represent this same $5 \mathrm{GHz}$ emission for comparison. The yellow contours show the outlines of the FellWalker identified cores. Bottom left panel: $\mathrm{H} 29 \alpha$ emission (black) with its Gaussian fit. The grey line shows the residuals from the Gaussian fit. In these spectra, the emission has been recentred to the expected rest velocity of the source, thus the velocity shift of the line peak (from zero) represents a difference between the ionised and molecular gas velocities. Top two panels on the right: moment 0 and moment 1 maps of the $\mathrm{H} 29 \alpha$ emission (top and bottom), while bottom two panels on the right: moment 0 and moment 1 maps of the CS emission (top and bottom).

correlation between regions. Around G302.02 the CS shows evidence for some kind of velocity gradient roughly east to west below the HiI region. For the other region with a suggestion of ionised infall (G336.98), in the $\mathrm{H} 29 \alpha$, there is no equivalent collimated structure in the CS. In both cases, however, the HII region does, again, appear to be near the edges of the molecular emission.

\section{Conclusions}

We have conducted a study of the mm emission from nine high-mass star-forming regions associated with young HII regions. We show that these regions are clustered (or at least clumpy) and that the separations between cores are more consistent with the thermal Jeans lengths than the turbulent Jeans 

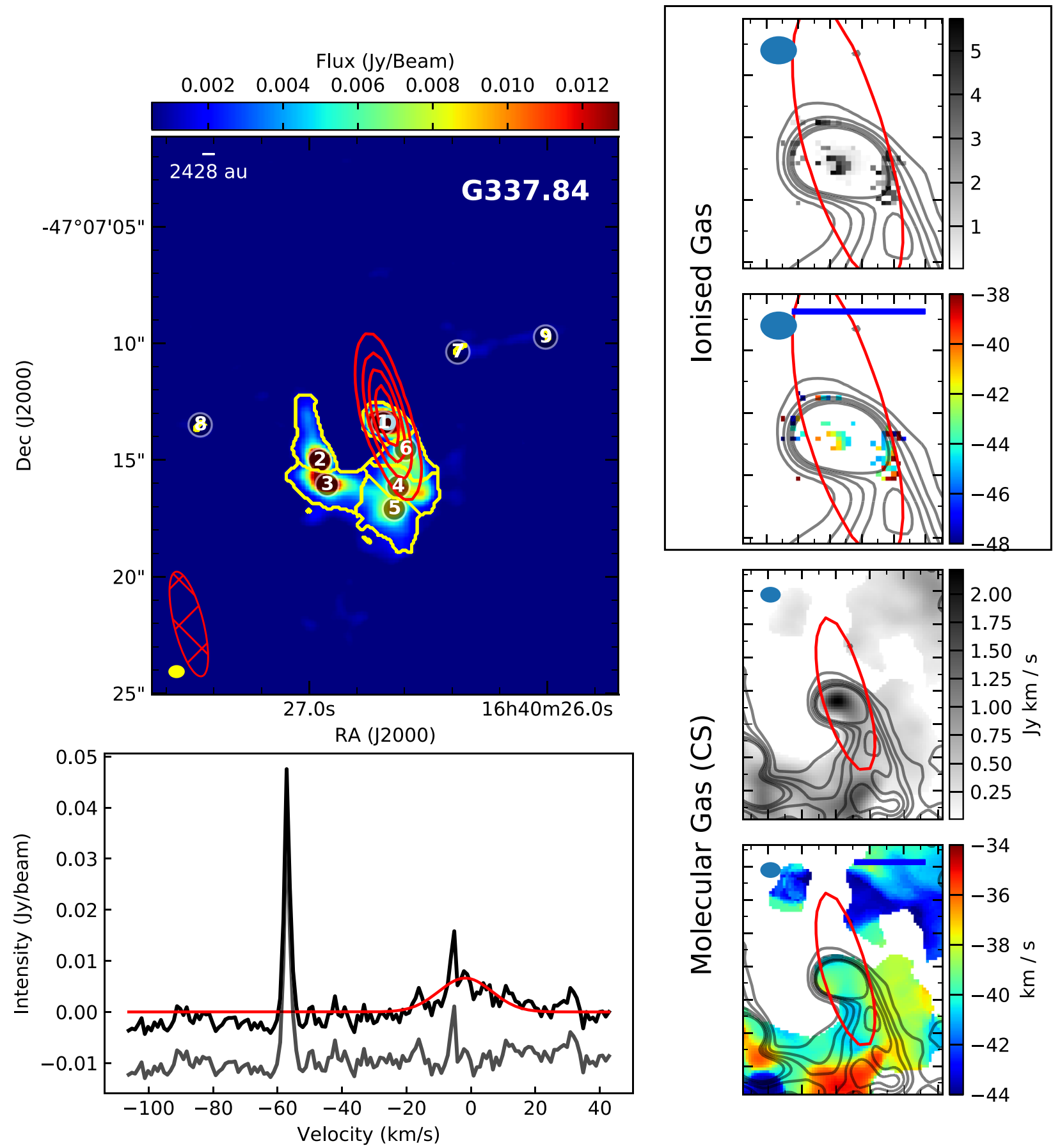

Fig. 5. Continuum, $\mathrm{H} 29 \alpha$, and CS emission from G337.84. Top left panel: $256 \mathrm{GHz}$ continuum in colour scale in which the $5 \mathrm{GHz}$ emission is shown in red contours; the red contours in the other images represent this same $5 \mathrm{GHz}$ emission for comparison. The yellow contours show the outlines of the FellWalker identified cores. Bottom left panel: $\mathrm{H} 29 \alpha$ emission (black) with its Gaussian fit. The grey line shows the residuals from the Gaussian fit. In these spectra, the emission has been recentred to the expected rest velocity of the source, thus the velocity shift of the line peak (from zero) represents a difference between the ionised and molecular gas velocities. Top two panels on the right: moment 0 and moment 1 maps of the $\mathrm{H} 29 \alpha$ emission (top and bottom), while bottom two panels on the right: moment 0 and moment 1 maps of the CS emission (top and bottom).

lengths given the pre-cursor densities and temperatures of the cores. We determined the masses of each of the identified cores, and find that, at most, a few of these cores in each region have masses great enough to provide enough mass for a high-mass star, if they have low temperatures.

We find evidence for $\mathrm{H} 29 \alpha$ emission in six out of nine regions, and that, in many of these regions, the $\mathrm{H} 29 \alpha$ spectrum is contaminated by molecular emission that needs to be disentangled to properly quantify the RRL emission. None of the $\mathrm{H} 29 \alpha$ profiles show any indications of pressure broadening, which puts upper limits on the electron densities of these HII regions at roughly $10^{7} \mathrm{~cm}^{-3}$. The HII regions with $\mathrm{H} 29 \alpha$ detections can broadly be separated into two types: those defined spatially as cometary (G330.28 and G337.63), and those defined by their bulls-eye dynamics (G302.02, G336.98, and potentially G339.11) indicative of infalling motions. 

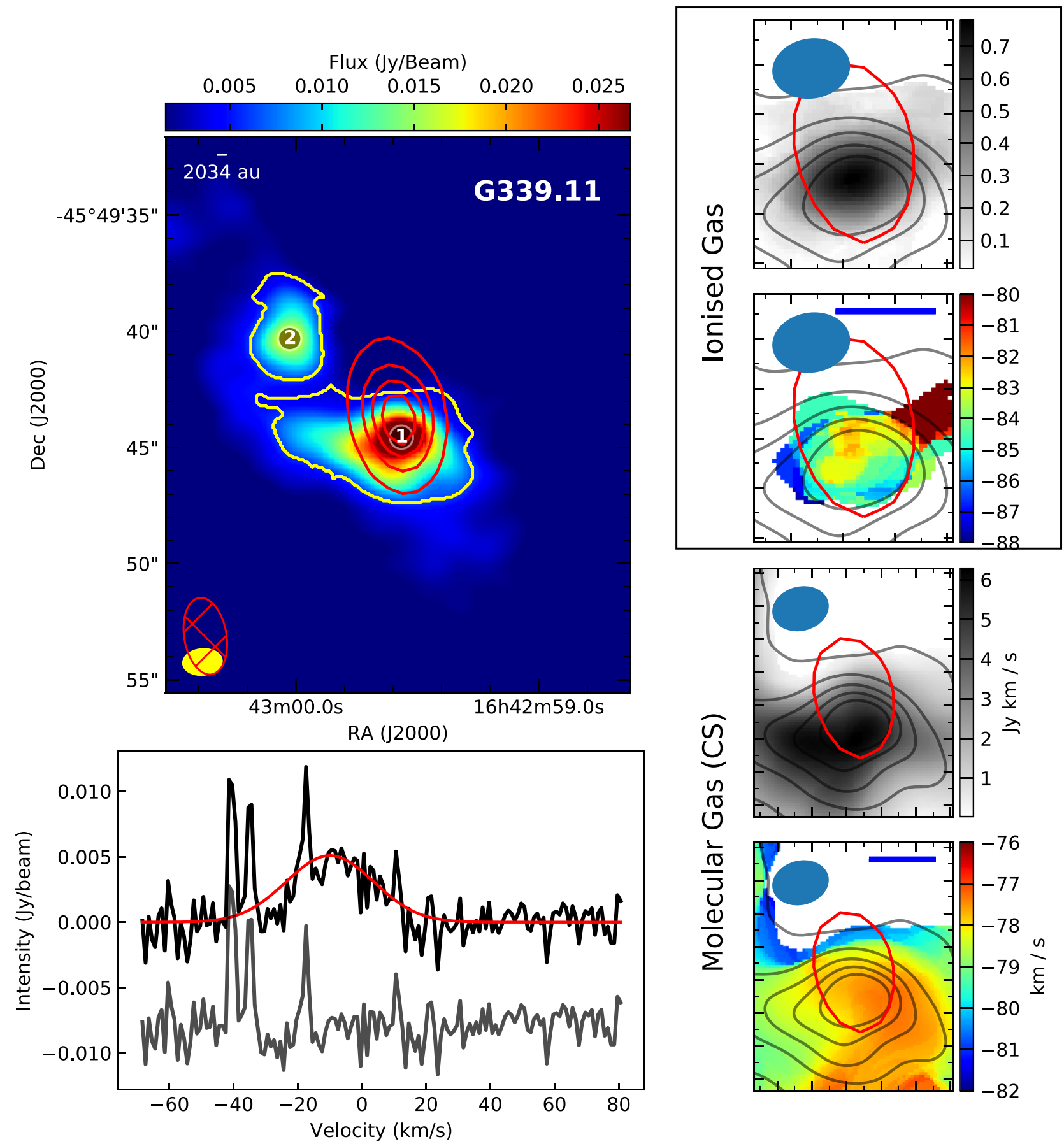

Fig. 6. Continuum, $\mathrm{H} 29 \alpha$, and CS emission from G339.11. Top left panel: $256 \mathrm{GHz}$ continuum in colour scale in which the $5 \mathrm{GHz}$ emission is shown in red contours; the red contours in the other images represent this same $5 \mathrm{GHz}$ emission for comparison. The yellow contours show the outlines of the FellWalker identified cores. Bottom left panel: H29 $\alpha$ emission (black) with its Gaussian fit. The grey line shows the residuals from the Gaussian fit. In these spectra, the emission has been recentred to the expected rest velocity of the source, thus the velocity shift of the line peak (from zero) represents a difference between the ionised and molecular gas velocities. Top two panels on the right: moment 0 and moment 1 maps of the $\mathrm{H} 29 \alpha$ emission (top and bottom), while bottom two panels on the right: moment 0 and moment 1 maps of the CS emission (top and bottom).

Of our sample of nine, the regions expected to have the youngest HII regions (from their radio spectral indices) show evidence for infall motions in the H29 $\alpha$ emission. This could be indicative of late stage accretion onto the central star(s) despite the presence of HII regions. In these cases, we find evidence both for the infalling HII region to be shrouded in its chemically rich hot core, and for this infalling region to have emerged from this hot core. For those $\mathrm{H} 29 \alpha$ detections that appear cometary (both morphologically and in their velocities), we find a much stronger correlation with the emission of the surrounding molecular gas; there is very little molecular gas at the tail and there is a bow in the molecular gas ahead of the HII region. The question of whether the bow of molecular emission is caused by the HII region moving through the medium or is the reason the cometary morphology exists (e.g. it is a champagne flow through a low density cavity) requires further study and comparison with models to disentangle. 

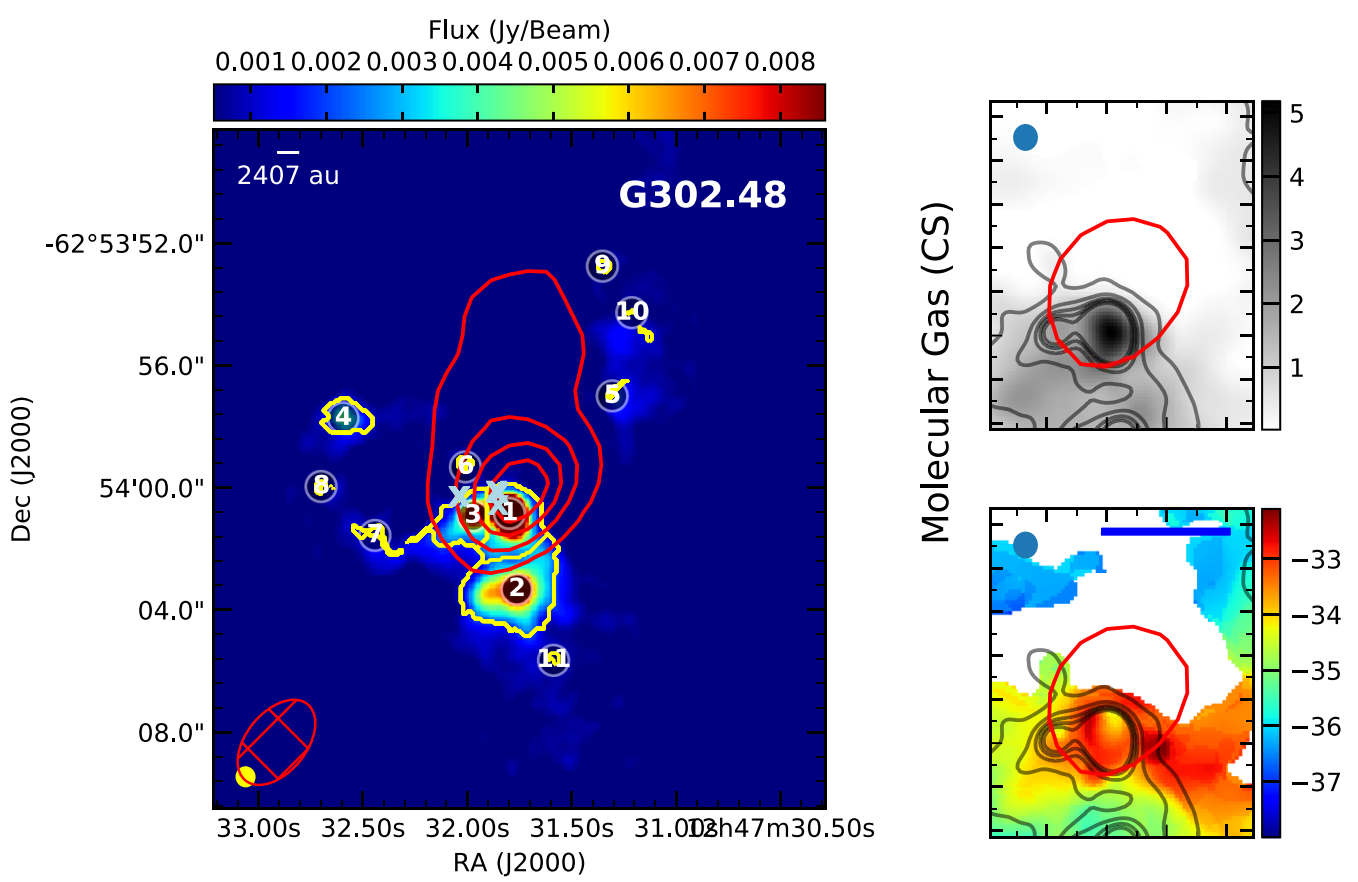

Fig. 7. Continuum and CS emission from G302.48. Left panel: $256 \mathrm{GHz}$ continuum in colour scale with $5 \mathrm{GHz}$ emission in red contours. The yellow contours show the outlines of the FellWalker identified cores. Two right panels: moment 0 and moment 1 (top and bottom, respectively) maps of the CS emission focussed on the brightest clump, where the HII region is expected to be (although no H29 $\alpha$ was detected, the $5 \mathrm{GHz}$ continuum shows its location).
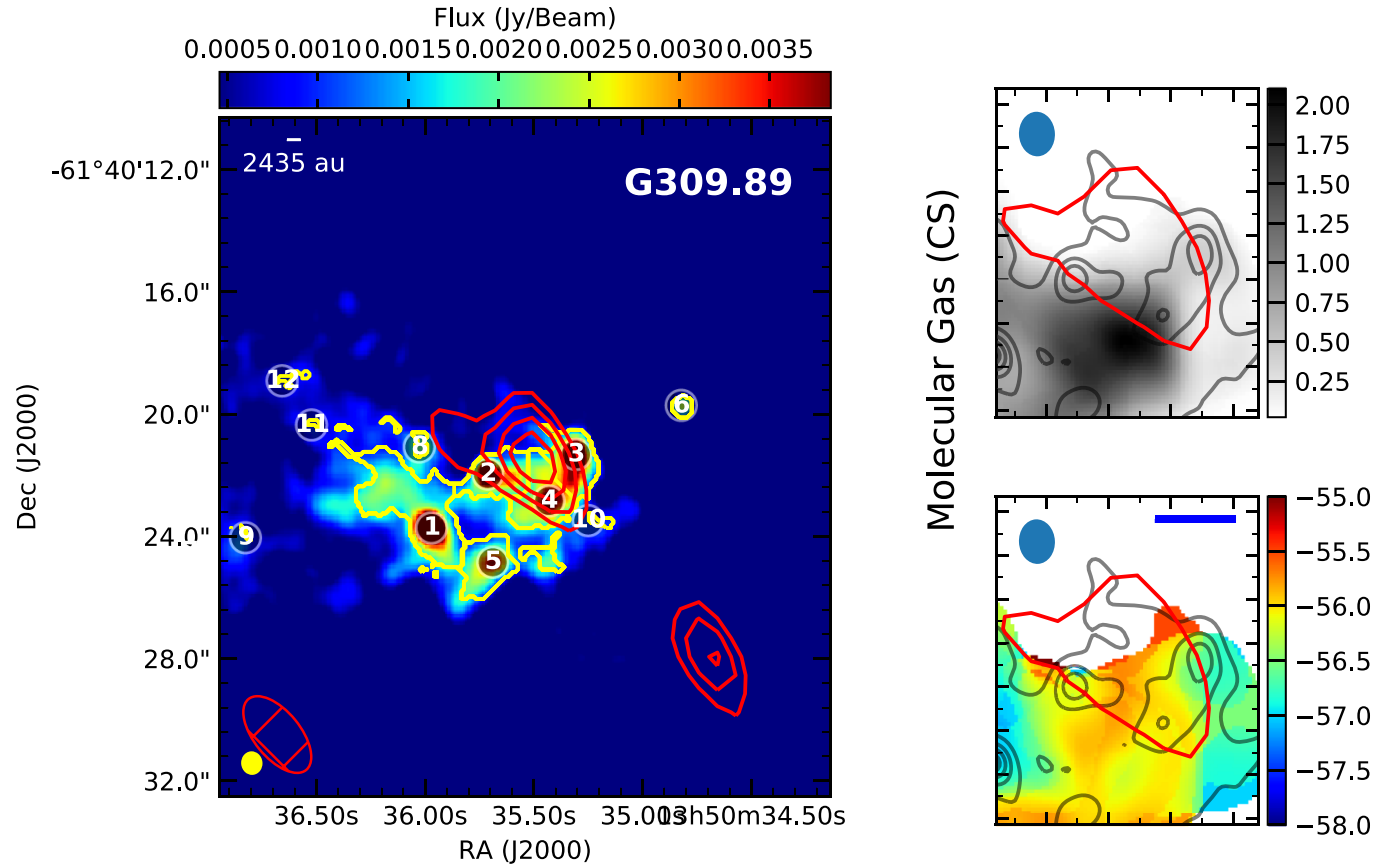

Fig. 8. Continuum and CS emission from G309.89. Left panel: $256 \mathrm{GHz}$ continuum in colour scale with $5 \mathrm{GHz}$ emission in red contours. The yellow contours show the outlines of the FellWalker identified cores. Two right panels: moment 0 and moment 1 (top and bottom, respectively) maps of the CS emission focussed on the brightest clump, where the HII region is expected to be (although no $\mathrm{H} 29 \alpha$ was detected, the $5 \mathrm{GHz}$ continuum shows its location).

Acknowledgements. This research made use of NASA's Astrophysics Data System; the SIMBAD database, operated at CDS, Strasbourg, France; APLpy, an open-source plotting package for Python hosted at http://aplpy.github.com; Astropy, a community-developed core Python package for Astronomy (Astropy Collaboration et al. 2013); pyspeckit, an open-source spectral analysis and plotting package for Python hosted at http://pyspeckit.bitbucket.org. This paper makes use of the following ALMA data: ADS/JAO.ALMA\#2013.1.00327.S. ALMA is a partnership of ESO (representing its member states), NSF (USA), and NINS (Japan), together with NRC (Canada), NSC and ASIAA (Taiwan), and KASI (Republic of Korea), in cooperation with the Republic of Chile. The Joint ALMA Observatory is operated by ESO, AUI/NRAO and NAOJ. JCM and HB acknowledge support from the European Research Council under the European Community's Horizon 2020 framework programme (2014-2020) via the ERC Consolidator grant 'From Cloud to Star Formation (CSF)' (project number 648505). TP acknowledges the Deutsche Forschungsgemeinschaft (DFG) for funding through the SPP 1573, "The Physics of the Interstellar Medium". RK acknowledges financial support via the Emmy Noether Research Group on Accretion Flows and Feedback in Realistic Models of Massive Star Formation funded by the German Research Foundation (DFG) under grant no. KU 2849/3-1. 
P. D. Klaassen et al.: The evolution of young HII regions. I.
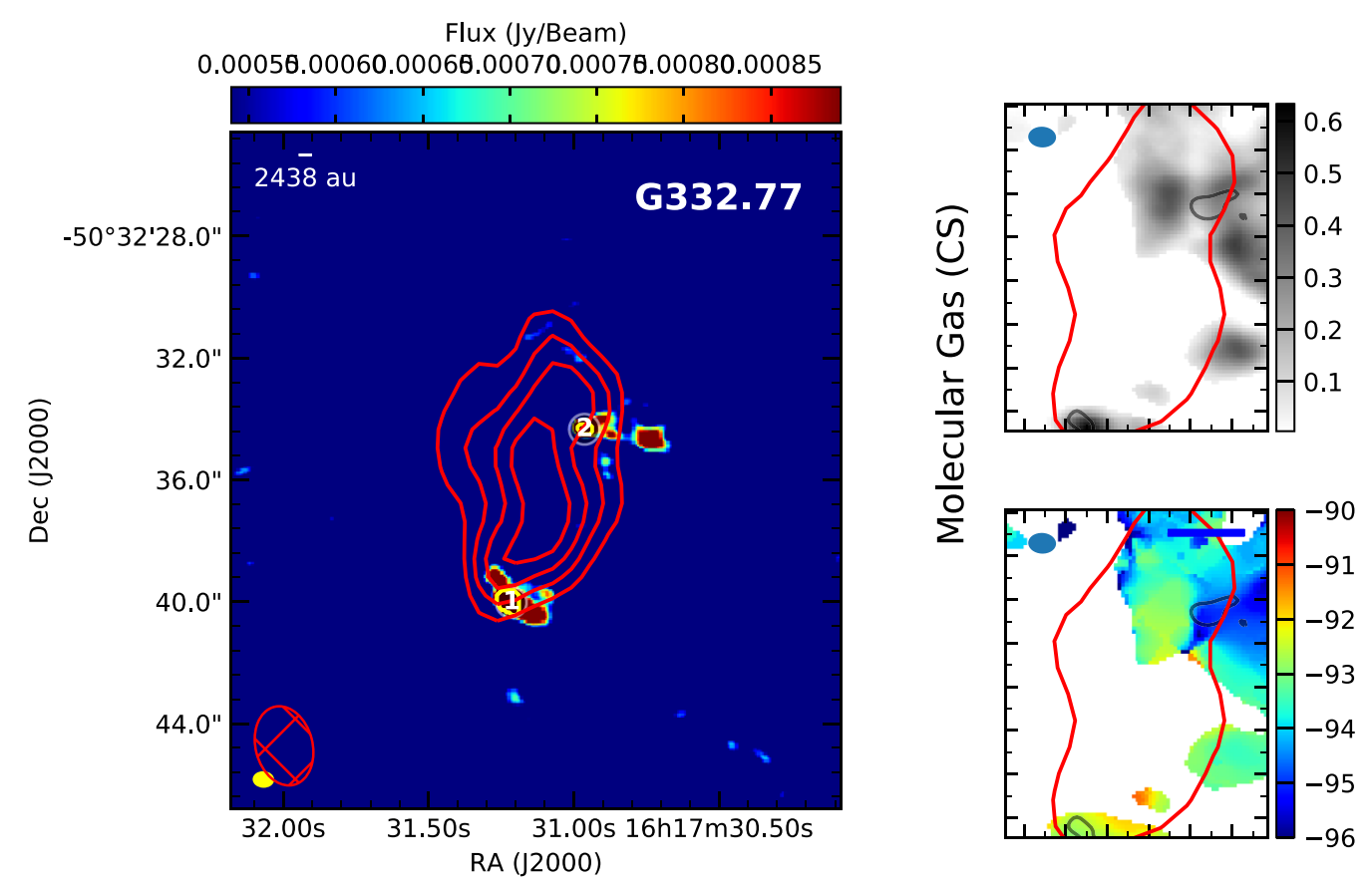

Fig. 9. Continuum and CS emission from G332.77. Left panel: $256 \mathrm{GHz}$ continuum in colour scale with $5 \mathrm{GHz}$ emission in red contours. The yellow contours show the outlines of the FellWalker identified cores. Two right panels: moment 0 and moment 1 (top and bottom, respectively) maps of the CS emission focussed on the brightest clump, where the HII region is expected to be (although no H29 $\alpha$ was detected, the $5 \mathrm{GHz}$ continuum shows its location).
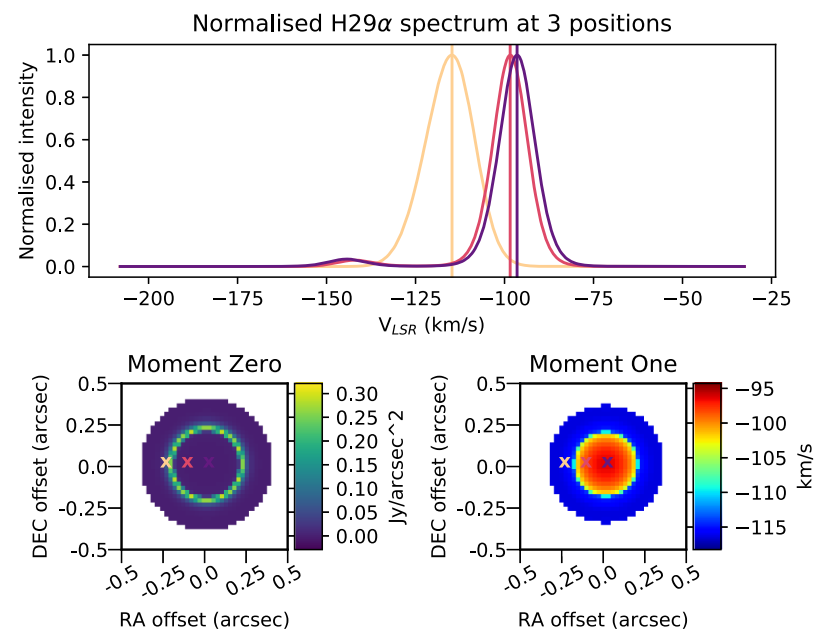

Fig. 10. Modelled H29 $\alpha$ emission coming from an infalling HII region. Model taken from Peters et al. (2012) and rerun for the H29 $\alpha$ transition.

\section{References}

Allison, R. J., Goodwin, S. P., Parker, R. J., et al. 2009, MNRAS, 395, 1449 Astropy Collaboration, Robitaille, T. P., Tollerud, E. J., et al. 2013, A\&A, 558, A33

Berry, D. S. 2015, Astron. Comput., 10, 22

Brocklehurst, M. \& Seaton, M. J. 1972, MNRAS, 157, 179

Brown, R. L., Lockman, F. J., \& Knapp, G. R. 1978, ARA\&A, 16, 445

Caswell, J. L. \& Haynes, R. F. 1987, A\&A, 171, 261

Csengeri, T., Leurini, S., Wyrowski, F., et al. 2016, A\&A, 586, A149

Davies, B., Hoare, M. G., Lumsden, S. L., et al. 2011, MNRAS, 416, 972

De Pree, C. G., Peters, T., Mac Low, M. M., et al. 2014, ApJ, 781, L36

De Pree, C. G., Peters, T., Mac Low, M. M., et al. 2015, ApJ, 815, 123

Draine, B. T. 2004, The Cold Universe, Saas-Fee Advanced Course 32, Lect. Notes 2002 of the Swiss Society for Astronomy and Astrophysics (SSAA), Springer-Verlag, eds A. W. Blain, F. Combes, B. T. Draine, D. Pfenniger, \& Y. Revaz, 213

Galván-Madrid, R., Goddi, C., \& Rodríguez, L. F. 2012, A\&A, 547, L3 Gordon, M. A. \& Sorochenko, R. L. 2002, Radio Recombination Lines. Their Physics and Astronomical Applications, 282

Haemmerlé, L. \& Peters, T. 2016, MNRAS, 458, 3299

Hildebrand, R. H. 1983, QJRAS, 24, 267

Hoare, M. G., \& Franco, J. 2007, Proc. Astrophys. Space Sci., 1, 61

Hoare, M. G., Kurtz, S. E., Lizano, S., Keto, E., \& Hofner, P. 2007, Protostars and Planets V, 181

Hosokawa, T., Yorke, H. W., \& Omukai, K. 2010, ApJ, 721, 478

Immer, K., Cyganowski, C., Reid, M. J., \& Menten, K. M. 2014, A\&A, 563, A39

Kauffmann, J., Bertoldi, F., Bourke, T. L., Evans, N. J. I., \& Lee, C. W. 2008, A\&A, 487, 993

Kennicutt, R. C. \& Evans, N. J. 2012, ARA\&A, 50, 531

Keto, E. \& Wood, K. 2006, ApJ, 637, 850

Keto, E., Zhang, Q., \& Kurtz, S. 2008, ApJ, 672, 423

Klassen, M., Peters, T., \& Pudritz, R. E. 2012, ApJ, 758, 137

Kuiper, R. \& Yorke, H. W. 2013, ApJ, 772, 61

Kuiper, R., Turner, N. J., \& Yorke, H. W. 2016, ApJ, 832, 40

Kurtz, S. 2005, Astrochemistry: Recent Successes and Current Challenges, 231, 47

Lumsden, S. L., Hoare, M. G., Urquhart, J. S., et al. 2013, ApJS, 208, 11

McMullin, J. P., Waters, B., Schiebel, D., Young, W., \& Golap, K. 2007, in Astronomical Data Analysis Software and Systems XVI, eds. R. A. Shaw, F. Hill, \& D. J. Bell, ASP Conf. Ser., 376, 127

Möller, T., Endres, C., \& Schilke, P. 2017, A\&A, 598, A7

Mottram, J. C., Hoare, M. G., Davies, B., et al. 2011a, ApJ, 730, L33

Mottram, J. C., Hoare, M. G., Urquhart, J. S., et al. 2011b, A\&A, 525, A149

Peters, T., Longmore, S. N., \& Dullemond, C. P. 2012, MNRAS, 425, 2352

Puls, J., Vink, J. S., \& Najarro, F. 2008, A\&ARv, 16, 209

Purser, S. J. D., Lumsden, S. L., Hoare, M. G., et al. 2016, MNRAS, 460, 1039

Reid, M. J., Dame, T. M., Menten, K. M., \& Brunthaler, A. 2016, ApJ, 823, 77

Sollins, P. K., Zhang, Q., Keto, E., \& Ho, P. T. P. 2005, ApJ, 624, L49

Urquhart, J. S., Busfield, A. L., Hoare, M. G., et al. 2007, A\&A, 461, 11

Urquhart, J. S., Hoare, M. G., Purcell, C. R., et al. 2009, A\&A, 501, 539

Urquhart, J. S., Thompson, M. A., Moore, T. J. T., et al. 2013, MNRAS, 435, 400

Urquhart, J. S., Moore, T. J. T., Csengeri, T., et al. 2014, MNRAS, 443, 1555

VanderPlas, J., Connolly, A. J., Ivezić, Ž., \& Gray, A. 2012, in Proc. Conf. Intelligent Data Understanding (CIDU), 47

Wang, K., Zhang, Q., Testi, L., et al. 2014, MNRAS, 439, 3275

Zapata, L. A., Leurini, S., Menten, K. M., et al. 2008, AJ, 136, 1455 


\section{Appendix A: Capturing the flux on large scales}

\section{A.1. Combining multi-configuration datasets}

For the continuum datasets, the relevant (i.e. line free) channels were averaged together to speed processing, and then the measurement sets from the individual array configurations were concatenated together. This single concatenated dataset was then Fourier transformed into the image plane using the clean command. Similarly, for the line datasets, we found that splitting out the relevant channels of the given spectral window (i.e. the channels within $100 \mathrm{~km} \mathrm{~s}^{-1}$ of the rest velocity), concatenating the configurations, and inverting the concatenated dataset gave the best signal-to-noise ratios in the final image plane maps.

As described further below, we quantified whether the ACA data was required for imaging. In terms of $\mathrm{H} 29 \alpha$ emission, we found no significant differences $(<1 \sigma)$ in the emission profiles between combining only $12 \mathrm{~m}$ configuration data and combining them with the ACA data as well in the target with the brightest H29 $\alpha$ emission (G302.02). For the continuum emission, we found that more flux was recovered when incorporating the ACA data. Comparing the integrated fluxes in our $26^{\prime \prime}$ primary beam to those from the ATLASGAL $350 \mathrm{GHz}$ emission (averaged over a $35^{\prime \prime}$ beam), we found that with the combination of $12 \mathrm{~m}$ and ACA observations, we recovered all of the flux in these regions with our observations, taking into account the different frequencies of the observations. The largest angular scale to which our observations were sensitive was comparable to our primary beam, and our achieved synthesised beams and sensitivities are listed in Table 1.

G302.02 appears to be the target with both the largest scale continuum and $\mathrm{H} 29 \alpha$ emission, and so to show that we have captured all of the flux for all of our targets, we use this region as an example to show how well the data combinations worked.

\section{A.2. Continuum emission}

The ACA only images of the continuum emission showed that the continuum was resolved, suggesting that the $12 \mathrm{~m}$ data alone would be insufficient to capture the full continuum flux. The left panels of Fig. A.1 show the continuum emission captured by the two 12 m configurations and that of the ACA for G302.02, which is the target with the most extended emission. The right panels of Fig. A.1 shows the combination of $12 \mathrm{~m}$ only data (bottom), and combination of $12 \mathrm{~m}$ and ACA data (top) for comparison. The colour scale is the same for all panels, shown with logarithmic scaling to highlight low-lying emission, and is tied to the peak flux of the fully combined (12 m + ACA) flux scale. The peak flux densities in the two right panels (of the combined data) are very similar (to within 3 sigma of each other at 24.1 and $23.9 \mathrm{mJy}$ beam $^{-1}$ for the full and $12 \mathrm{~m}$ only data, respectively), however in the $12 \mathrm{~m}$ only dataset, we recover only $80 \%$ of the flux present in the full (i.e. including ACA) dataset.

Figure A. 2 shows how the amplitude of the emission varies as a function of uv distance. Of note here is the overlapping uv distances of the three configurations and generally consistent fluxes between configurations. The data are sampled well enough in the overlap region to allow for proper combination of the datasets.

\section{A.3. H29 $\alpha$ emission}

H29 $\alpha$ was detected in most of our sources and on small enough scales to not require the use of ACA data. It was only detected in the stand-alone ACA data for G302.02, which is the target with the brightest and most extended $\mathrm{H} 29 \alpha$ emission. However, as shown in Fig. A.3, the $12 \mathrm{~m}$ array data recover all the flux so it was not necessary to combine this data with the ACA data.

\section{Appendix B: lonised and molecular gas separation}

\section{B.1. XCLASS modelling}

HII regions form inside the dense envelopes of MYSOs, often generating or enlarging the region of chemically rich molecular emission, a so-called hot-core. As such, spectroscopic studies of the youngest HII regions often need to deal with blending of ionised and molecular gas features in the spectrum. This is the case for many (but not all) of the HII regions in this study. G336.98, amongst the targets with $\mathrm{H} 29 \alpha$ detections, had the strongest molecular signatures, and is used here to illustrate the separation techniques employed in this study (see Fig. B.1).

As can be seen in the top panel of Fig. B.1, a number of molecular lines overlap in frequency with the much broader RRL emission. The primary sources of these spectral features are $\mathrm{CH}_{3} \mathrm{CCH}$ (five lines), $\mathrm{CH}_{3} \mathrm{OCHO}$, and in some cases, methanol and ethanol. We only fit $\mathrm{CH}_{3} \mathrm{CCH}$ in $\mathrm{G} 336.98$ because its multiple components allowed us to place greater constraints on the rest velocities and relative intensities of the emission lines. Models with other species that had less constraints on the parameter space failed to converge on repeatable solutions.

Here, we describe the steps involved in fitting the various species, and how we separated the ionised and molecular species for analysis. The analysis was performed on smaller subregions within each observed field of view. This subregion corresponds to the region bounded by the continuum core containing the H29 $\alpha$ emission or, in its absence, the brightest core. The exception to this rule was G337.63, where the fourth brightest core contained the $\mathrm{H} 29 \alpha$ emission.

To ensure our species fitting had a physical basis rather than producing a (potentially) disjointed set of independent Gaussians that could change dramatically from pixel to pixel, we used the LTE version of XCLASS (version 1.2) to fit our spectra (Möller et al. 2017). In this process, we found that if the $\mathrm{H} 29 \alpha$ emission were fit in combination with the molecules, the removal of the molecular species would be relatively straightforward. However, testing showed that in not fitting the $\mathrm{H} 29 \alpha$ emission, the resulting spectra (after having removed the poorly fit molecules) had large absorption features in the remaining $\mathrm{H} 29 \alpha$ spectrum because the intensities of the molecular species had been over estimated to fit the spectra.

RRLs are not included in the databases compatible with XCLASS, which necessitated having to add the $n=29$ transition of hydrogen to the local copy of the database. A new entry was made into the sqlite 3 database with a transition frequency corresponding to that of $\mathrm{H} 29 \alpha$, and dummy values for the components of the required partition function. In this way, XCLASS could simultaneously fit the molecules and H29 $\alpha$ emission, avoiding problems with overestimating the molecular emission at $\mathrm{H} 29 \alpha$ frequencies. In no way are the fitted physical properties (i.e. $\mathrm{N}$ and T) of the $\mathrm{H} 29 \alpha$ emission derived from the fitted spectra realistic because the partition function used to derive the physical properties is unrealistic (i.e. populated with zeros). However, the Gaussian properties of the fit (i.e. FWHM and $v_{\max }$ ) are realistic as they are empirically fit to the data along with the molecular species. The physical properties derived from the 
P. D. Klaassen et al.: The evolution of young Hil regions. I.

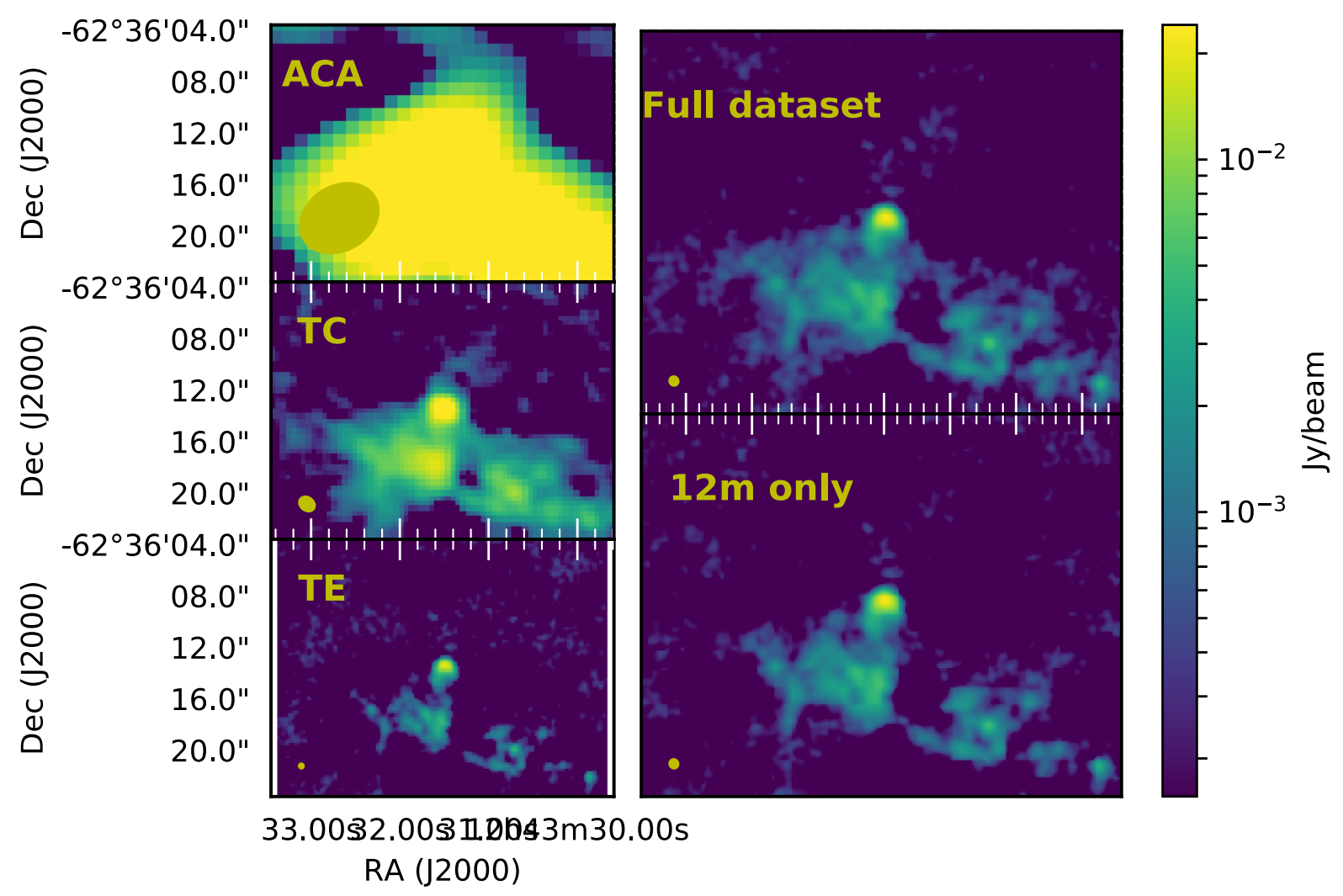

Fig. A.1. Continuum data combination for G302.02: Left three panels: data obtained with each individual antenna configuration; the two 12 $\mathrm{m}$ configurations are labelled as TC and TE for the compact and extended configurations, respectively. Right panels: combined datasets. Bottom right panel: $12 \mathrm{~m}$ data only, while top right panel: full continuum dataset. The integrated fluxes of the two right panels vary by $20 \%$, although their peak fluxes are relatively consistent with each other. The scaling is the same for all five plots, as shown at the right.

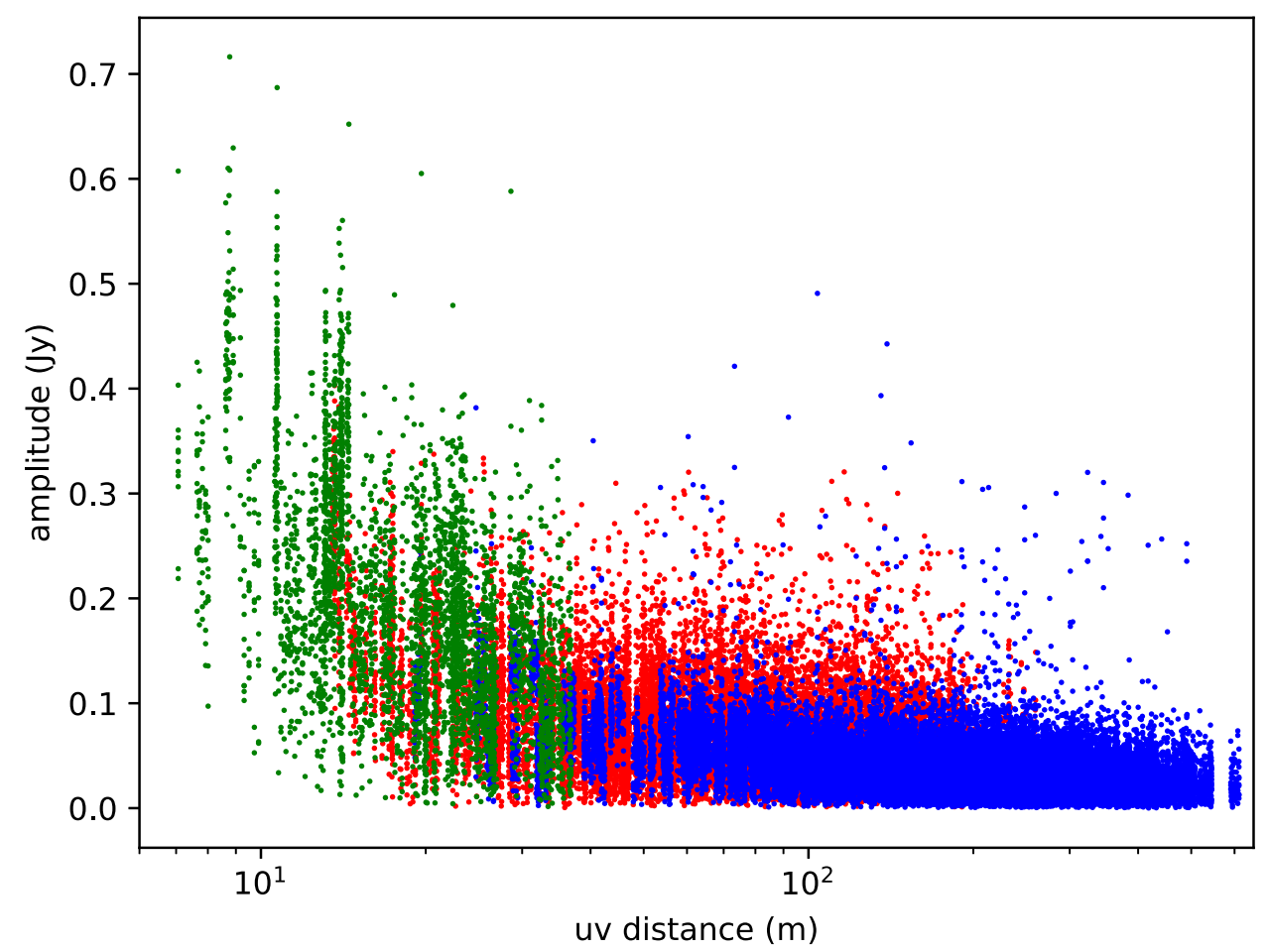

Fig. A.2. Continuum emission in the three configurations: ACA (green), compact $12 \mathrm{~m}$ (red), and extended $12 \mathrm{~m}$ (blue). The amplitudes are generally consistent with each other, given the rms noise limits in each map, and show the same trends with uv distance (e.g. higher amplitudes at lower spatial frequencies - larger size scales). 
Table A.1. Calibrators and Observing Parameters for ALMA project 2013.1.00327.S.

\begin{tabular}{|c|c|c|c|c|c|c|c|}
\hline \multirow[b]{2}{*}{ Execution $^{a}$} & \multirow{2}{*}{$\begin{array}{l}\text { Time on each } \\
\text { Science source } \\
\text { (min) }\end{array}$} & \multicolumn{3}{|c|}{ Calibrators } & \multirow[b]{2}{*}{ Observing date } & \multicolumn{2}{|c|}{ Baseline lengths } \\
\hline & & Phase & Bandpass & Flux & & $\begin{array}{l}\text { Max } \\
(\mathrm{m})\end{array}$ & $\begin{array}{l}\text { Min } \\
(\mathrm{m})\end{array}$ \\
\hline \multicolumn{8}{|c|}{ Targets near G305 (3 regions) } \\
\hline \multicolumn{8}{|c|}{ extended 12m configuration $(\mathrm{pwv}=1.45 \mathrm{~mm})$} \\
\hline $\mathrm{X} 230$ & 9 & J1254-6111 & J1427-4206 & J1107-448 & $2015-06-04$ & 784 & 21.4 \\
\hline \multicolumn{8}{|c|}{ compact $12 \mathrm{~m}$ configuration $(\mathrm{pwv}=3.82 \mathrm{~mm})$} \\
\hline $\mathrm{X} 2474$ & 4 & $\mathrm{~J} 1254-6111$ & $\mathrm{~J} 1107-4449$ & Callisto & $2015-01-15$ & 349 & 15.1 \\
\hline \multicolumn{8}{|c|}{$\mathbf{A C A}(\mathrm{pwv}=1.52 \mathrm{~mm})$} \\
\hline X610 & 8 & J1308-6707 & $\mathrm{J} 1427-4206$ & Titan & 2014-06-07 & 48 & 9.1 \\
\hline X1ff & 8 & J1308-6707 & J1427-4206 & Titan & 2014-06-07 & 48 & 9.1 \\
\hline X610 & 8 & J1308-6707 & J1427-4206 & Titan & 2014-06-07 & 48 & 9.1 \\
\hline X1ff & 8 & J1308-6707 & J1427-4206 & Titan & 2014-06-07 & 48 & 9.1 \\
\hline
\end{tabular}

Targets near G335 (6 regions)

\begin{tabular}{|c|c|c|c|c|c|c|c|}
\hline \multicolumn{8}{|c|}{ extended $12 \mathrm{~m}$ configuration $(\mathrm{pwv}=1.36 \mathrm{~mm})$} \\
\hline $\mathrm{X} 15 \mathrm{f} 4$ & 8 & J1617-5848 & J1617-5848 & Titan & $2015-06-04$ & 784 & 21.4 \\
\hline \multicolumn{8}{|c|}{ compact $12 \mathrm{~m}$ configuration $(\mathrm{pwv}=1.96 \mathrm{~mm})$} \\
\hline X2ade & 4 & J1617-5848 & J1617-5848 & Ceres & $2015-01-03$ & 349 & 15.1 \\
\hline \multicolumn{8}{|c|}{$\mathbf{A C A}(\mathrm{pwv}=0.84 \mathrm{~mm})$} \\
\hline X6823 & 5 & J1617-5848 & $\mathrm{J} 1427-4206$ & Titan & 2015-06-07 & 49 & 8.9 \\
\hline $\mathrm{X} 1 \mathrm{f} 1$ & 5 & J1617-5848 & J1617-5848 & J1613-586 & 2014-07-29 & 49 & 8.9 \\
\hline $\mathrm{X} 1804^{b}$ & 1 & $\mathrm{~J} 1617-5848$ & J1617-5848 & Titan & 2015-04-22 & 48 & 9.1 \\
\hline $\mathrm{Xd} 0$ & 5 & J1617-5848 & J1617-5848 & Mars & 2014-07-27 & 49 & 8.9 \\
\hline Xa6e & 5 & J1617-5848 & $\mathrm{J} 1617-5848$ & J1613-586 & 2014-07-28 & 49 & 8.9 \\
\hline $\mathrm{X} 1 \mathrm{e} 58$ & 5 & J1617-5848 & J1924-2914 & Titan & $2015-06-03$ & 49 & 8.9 \\
\hline $\mathrm{X} 1 \mathrm{ae} 0$ & 5 & J1617-5848 & J1617-5848 & Titan & 2015-06-03 & 91 & 8.9 \\
\hline
\end{tabular}

Notes. ${ }^{(a)}$ The execution ID is the end portion of the unique filename given to every ALMA observation. ${ }^{(b)}$ G336.98 and G339.11 were not included in this execution.

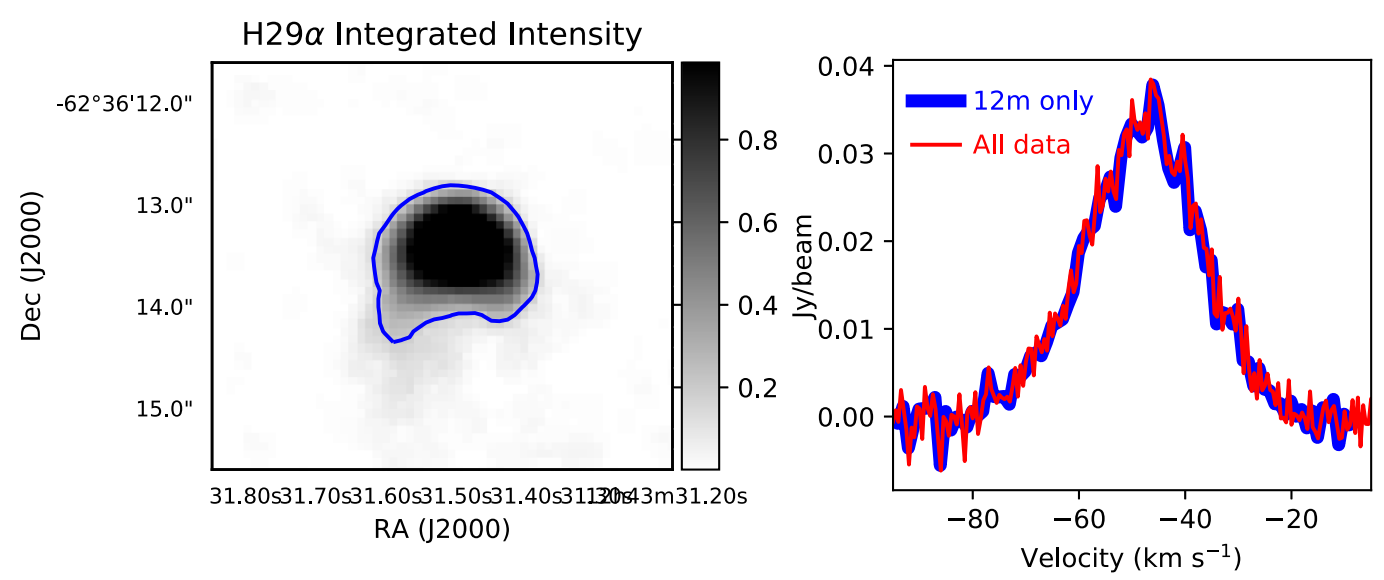

Fig. A.3. H29 $\alpha$ Data combination: Comparison between $12 \mathrm{~m}$ only and full dataset for capturing the $\mathrm{H} 29 \alpha$ flux. Left: integrated intensity of $\mathrm{H} 29 \alpha$ towards G302.02, where the blue contour shows the $10 \%$ power contour of the emission. The map was created with a $3 \sigma$ clipping mask and the greyscale is limited to 50\% power to highlight the low-lying emission. Right: H29 $\alpha$ spectra integrated within the $10 \%$ contour shown on the left. The blue (thick) line shows the $12 \mathrm{~m}$ only data, while the red (thin) line shows the combination of $12 \mathrm{~m}$ and ACA data. The lack of significant differences between the spectra suggests that the ACA is not required to capture all of the flux from the RRLs. 
P. D. Klaassen et al.: The evolution of young HII regions. I.

Table A.2. Clump positions, areas, derived fluxes, masses, and column densities.

\begin{tabular}{|c|c|c|c|c|c|c|c|c|c|c|}
\hline \multirow[t]{2}{*}{ ID number } & \multirow{2}{*}{$\begin{array}{c}\text { Peak RA } \\
\text { (h:m:s) }\end{array}$} & \multirow{2}{*}{$\begin{array}{c}\text { Peak Dec } \\
\text { (d:m:s) }\end{array}$} & \multirow{2}{*}{$\begin{array}{c}\text { Area } \\
\left(\operatorname{arcsec}^{2}\right)\end{array}$} & \multirow{2}{*}{$\begin{array}{l}\text { Flux } \\
(\mathrm{mJy})\end{array}$} & \multicolumn{3}{|c|}{ Clump mass $\left(M_{\odot}\right)$} & \multicolumn{3}{|c|}{ Log(Column density) } \\
\hline & & & & & $(20 \mathrm{~K})$ & $(50 \mathrm{~K})$ & $(100 \mathrm{~K})$ & $(20 \mathrm{~K})$ & $(50 \mathrm{~K})$ & $(100 \mathrm{~K})$ \\
\hline \multicolumn{11}{|c|}{$\mathrm{G} 302.02\left(1.00 \mathrm{e}+07 \mathrm{~cm}^{-3}, 70.0 \mathrm{~K}\right)$} \\
\hline 1 & $12: 43: 31.50$ & $-62: 36: 13.36$ & 4.63 & 62.08 & 25.37 & 8.37 & 3.94 & 24.41 & 23.93 & 23.6 \\
\hline 2 & $12: 43: 31.54$ & $-62: 36: 17.68$ & 13.45 & 81.48 & 33.29 & 10.99 & 5.17 & 23.79 & 23.31 & 22.98 \\
\hline 3 & $12: 43: 30.71$ & $-62: 36: 19.92$ & 5.22 & 23.92 & 9.77 & 3.23 & 1.52 & 23.77 & 23.29 & 22.96 \\
\hline 4 & $12: 43: 29.87$ & $-62: 36: 22.00$ & 0.94 & 4.57 & 1.87 & 0.62 & 0.29 & 23.62 & 23.14 & 22.81 \\
\hline 5 & $12: 43: 32.33$ & $-62: 36: 16.80$ & 0.75 & 3.01 & 1.23 & 0.41 & 0.19 & 23.45 & 22.97 & 22.64 \\
\hline 6 & $12: 43: 30.92$ & $-62: 36: 21.12$ & 1.5 & 6.01 & 2.46 & 0.81 & 0.38 & 23.43 & 22.95 & 22.62 \\
\hline 7 & $12: 43: 32.17$ & $-62: 36: 18.32$ & 1.39 & 5.57 & 2.28 & 0.75 & 0.35 & 23.41 & 22.92 & 22.6 \\
\hline 8 & $12: 43: 30.41$ & $-62: 36: 18.72$ & 0.38 & 1.29 & 0.53 & 0.17 & 0.08 & 23.32 & 22.84 & 22.51 \\
\hline 9 & $12: 43: 30.36$ & $-62: 36: 21.28$ & 0.3 & 0.83 & 0.34 & 0.11 & 0.05 & 23.18 & 22.7 & 22.37 \\
\hline 10 & $12: 43: 31.23$ & $-62: 36: 20.40$ & 0.16 & 0.43 & 0.17 & 0.06 & 0.03 & 23.12 & 22.64 & 22.31 \\
\hline 11 & $12: 43: 30.30$ & $-62: 36: 22.08$ & 0.09 & 0.23 & 0.09 & 0.03 & 0.01 & 23.09 & 22.61 & 22.28 \\
\hline 12 & $12: 43: 32.52$ & $-62: 36: 15.60$ & 0.2 & 0.52 & 0.21 & 0.07 & 0.03 & 23.07 & 22.59 & 22.26 \\
\hline 13 & $12: 43: 31.89$ & $-62: 36: 14.40$ & 0.11 & 0.27 & 0.11 & 0.04 & 0.02 & 23.04 & 22.56 & 22.23 \\
\hline \multicolumn{11}{|c|}{$\mathrm{G} 302.48\left(1.00 \mathrm{e}+07 \mathrm{~cm}^{-3}, 70.0 \mathrm{~K}\right)$} \\
\hline 1 & $12: 47: 31.80$ & $-62: 54: 0.84$ & 4.31 & 93.89 & 24.3 & 8.02 & 3.77 & 24.69 & 24.21 & 23.88 \\
\hline 2 & $12: 47: 31.76$ & $-62: 54: 3.32$ & 6.84 & 73.15 & 18.93 & 6.25 & 2.94 & 24.21 & 23.73 & 23.4 \\
\hline 3 & $12: 47: 31.97$ & $-62: 54: 0.92$ & 2.74 & 27.3 & 7.06 & 2.33 & 1.1 & 24.14 & 23.65 & 23.33 \\
\hline 4 & $12: 47: 32.59$ & $-62: 53: 57.72$ & 1.5 & 8.32 & 2.15 & 0.71 & 0.33 & 23.68 & 23.2 & 22.87 \\
\hline 5 & $12: 47: 31.31$ & $-62: 53: 57.00$ & 0.14 & 0.47 & 0.12 & 0.04 & 0.02 & 23.28 & 22.79 & 22.47 \\
\hline 6 & $12: 47: 32.01$ & $-62: 53: 59.32$ & 0.2 & 0.62 & 0.16 & 0.05 & 0.02 & 23.21 & 22.72 & 22.4 \\
\hline 7 & $12: 47: 32.44$ & $-62: 54: 1.56$ & 0.49 & 1.47 & 0.38 & 0.13 & 0.06 & 23.18 & 22.7 & 22.37 \\
\hline 8 & $12: 47: 32.70$ & $-62: 53: 59.96$ & 0.13 & 0.4 & 0.1 & 0.03 & 0.02 & 23.15 & 22.67 & 22.34 \\
\hline 9 & $12: 47: 31.35$ & $-62: 53: 52.76$ & 0.17 & 0.51 & 0.13 & 0.04 & 0.02 & 23.14 & 22.66 & 22.33 \\
\hline 10 & $12: 47: 31.21$ & $-62: 53: 54.28$ & 0.17 & 0.46 & 0.12 & 0.04 & 0.02 & 23.11 & 22.63 & 22.3 \\
\hline 11 & $12: 47: 31.59$ & $-62: 54: 5.64$ & 0.13 & 0.38 & 0.1 & 0.03 & 0.02 & 23.1 & 22.62 & 22.29 \\
\hline \multicolumn{11}{|c|}{$\mathrm{G} 309.89\left(1.00 \mathrm{e}+07 \mathrm{~cm}^{-3}, 70.0 \mathrm{~K}\right)$} \\
\hline 1 & $13: 50: 35.97$ & $-61: 40: 23.72$ & 7.9 & 36.28 & 23.91 & 7.89 & 3.71 & 23.88 & 23.4 & 23.07 \\
\hline 2 & $13: 50: 35.71$ & $-61: 40: 21.96$ & 3.0 & 14.7 & 9.68 & 3.2 & 1.5 & 23.71 & 23.23 & 22.9 \\
\hline 3 & $13: 50: 35.30$ & $-61: 40: 21.32$ & 3.01 & 16.85 & 11.1 & 3.66 & 1.72 & 23.68 & 23.2 & 22.87 \\
\hline 4 & $13: 50: 35.43$ & $-61: 40: 22.84$ & 3.95 & 19.35 & 12.75 & 4.21 & 1.98 & 23.58 & 23.1 & 22.77 \\
\hline 5 & $13: 50: 35.69$ & $-61: 40: 24.84$ & 3.05 & 12.47 & 8.22 & 2.71 & 1.27 & 23.52 & 23.04 & 22.72 \\
\hline 6 & $13: 50: 34.82$ & $-61: 40: 19.72$ & 0.45 & 1.48 & 0.97 & 0.32 & 0.15 & 23.33 & 22.85 & 22.52 \\
\hline 7 & $13: 50: 37.08$ & $-61: 40: 24.52$ & 0.65 & 1.99 & 1.31 & 0.43 & 0.2 & 23.31 & 22.83 & 22.5 \\
\hline 8 & $13: 50: 36.02$ & $-61: 40: 21.08$ & 0.51 & 1.59 & 1.05 & 0.35 & 0.16 & 23.27 & 22.79 & 22.46 \\
\hline 9 & $13: 50: 36.82$ & $-61: 40: 24.04$ & 0.1 & 0.2 & 0.13 & 0.04 & 0.02 & 22.99 & 22.51 & 22.18 \\
\hline 10 & $13: 50: 35.25$ & $-61: 40: 23.48$ & 0.28 & 0.58 & 0.38 & 0.13 & 0.06 & 22.97 & 22.48 & 22.16 \\
\hline 11 & $13: 50: 36.52$ & $-61: 40: 20.36$ & 0.13 & 0.26 & 0.17 & 0.06 & 0.03 & 22.96 & 22.48 & 22.15 \\
\hline 12 & $13: 50: 36.65$ & $-61: 40: 18.92$ & 0.32 & 0.64 & 0.42 & 0.14 & 0.07 & 22.95 & 22.47 & 22.14 \\
\hline \multicolumn{11}{|c|}{$\mathrm{G} 330.28\left(1.00 \mathrm{e}+07 \mathrm{~cm}^{-3}, 86.0 \mathrm{~K}\right)$} \\
\hline 1 & $16: 3: 43.15$ & $-51: 51: 46.40$ & 10.92 & 79.9 & 53.44 & 17.64 & 8.29 & 24.08 & 23.6 & 23.27 \\
\hline 2 & $16: 3: 43.23$ & $-51: 51: 49.68$ & 3.53 & 22.14 & 14.8 & 4.89 & 2.3 & 24.04 & 23.55 & 23.23 \\
\hline 3 & $16: 3: 43.10$ & $-51: 51: 47.76$ & 4.02 & 26.07 & 17.43 & 5.75 & 2.7 & 23.81 & 23.33 & 23.0 \\
\hline 4 & $16: 3: 44.13$ & $-51: 51: 45.36$ & 0.7 & 1.52 & 1.02 & 0.34 & 0.16 & 23.13 & 22.65 & 22.32 \\
\hline 5 & $16: 3: 43.88$ & $-51: 51: 53.12$ & 0.6 & 1.21 & 0.81 & 0.27 & 0.13 & 23.12 & 22.63 & 22.31 \\
\hline 6 & $16: 3: 43.82$ & $-51: 51: 51.44$ & 0.29 & 0.63 & 0.42 & 0.14 & 0.07 & 23.11 & 22.63 & 22.3 \\
\hline 7 & $16: 3: 43.84$ & $-51: 51: 46.32$ & 0.44 & 0.8 & 0.54 & 0.18 & 0.08 & 22.99 & 22.51 & 22.18 \\
\hline 8 & $16: 3: 44.42$ & $-51: 51: 44.64$ & 0.24 & 0.41 & 0.28 & 0.09 & 0.04 & 22.99 & 22.51 & 22.18 \\
\hline \multicolumn{11}{|c|}{$\mathrm{G} 332.77\left(1.00 \mathrm{e}+07 \mathrm{~cm}^{-3}, 70.0 \mathrm{~K}\right)$} \\
\hline 1 & $16: 17: 31.21$ & $-50: 32: 40.02$ & 0.57 & 3.55 & 2.57 & 0.85 & 0.4 & 23.61 & 23.13 & 22.8 \\
\hline 2 & $16: 17: 30.96$ & $-50: 32: 34.34$ & 0.19 & 0.72 & 0.52 & 0.17 & 0.08 & 23.27 & 22.79 & 22.46 \\
\hline \multicolumn{11}{|c|}{$\mathrm{G} 336.98\left(1.00 \mathrm{e}+07 \mathrm{~cm}^{-3}, 33.0 \mathrm{~K}\right)$} \\
\hline 1 & $16: 36: 12.41$ & $-47: 37: 58.06$ & 9.57 & 243.88 & 80.13 & 26.45 & 12.43 & 25.23 & 24.75 & 24.42 \\
\hline 2 & $16: 36: 12.63$ & $-47: 37: 57.50$ & 2.52 & 25.73 & 8.45 & 2.79 & 1.31 & 24.07 & 23.59 & 23.26 \\
\hline 3 & $16: 36: 12.51$ & $-47: 37: 56.70$ & 2.04 & 19.56 & 6.43 & 2.12 & 1.0 & 23.94 & 23.46 & 23.13 \\
\hline 4 & $16: 36: 12.09$ & $-47: 37: 58.38$ & 2.2 & 12.91 & 4.24 & 1.4 & 0.66 & 23.7 & 23.22 & 22.89 \\
\hline
\end{tabular}


Table A.2. continued.

\begin{tabular}{|c|c|c|c|c|c|c|c|c|c|c|}
\hline \multirow[t]{2}{*}{ ID number } & \multirow{2}{*}{$\begin{array}{c}\text { Peak RA } \\
\text { (h:m:s) }\end{array}$} & \multirow{2}{*}{$\begin{array}{l}\text { Peak Dec } \\
\text { (d:m:s) }\end{array}$} & \multirow{2}{*}{$\begin{array}{c}\text { Area } \\
\left(\operatorname{arcsec}^{2}\right)\end{array}$} & \multirow{2}{*}{$\begin{array}{l}\text { Flux } \\
(\mathrm{mJy})\end{array}$} & \multicolumn{3}{|c|}{ Clump mass $\left(M_{\odot}\right)$} & \multicolumn{3}{|c|}{$\log ($ Column density $)$} \\
\hline & & & & & $(20 \mathrm{~K})$ & $(50 \mathrm{~K})$ & $(100 \mathrm{~K})$ & $(20 \mathrm{~K})$ & $(50 \mathrm{~K})$ & $(100 \mathrm{~K})$ \\
\hline \multicolumn{11}{|c|}{$\mathrm{G} 337.63\left(1.00 \mathrm{e}+07 \mathrm{~cm}^{-3}, 99.0 \mathrm{~K}\right)$} \\
\hline 1 & $16: 38: 19.11$ & $-47: 4: 53.66$ & 4.26 & 78.86 & 15.77 & 5.2 & 2.45 & 24.72 & 24.23 & 23.91 \\
\hline 2 & $16: 38: 19.26$ & $-47: 4: 57.58$ & 3.67 & 26.27 & 5.25 & 1.73 & 0.81 & 24.1 & 23.62 & 23.29 \\
\hline 3 & $16: 38: 19.09$ & $-47: 4: 52.70$ & 1.5 & 16.72 & 3.34 & 1.1 & 0.52 & 24.07 & 23.59 & 23.26 \\
\hline 4 & $16: 38: 19.09$ & $-47: 4: 51.18$ & 6.62 & 53.07 & 10.61 & 3.5 & 1.65 & 23.93 & 23.45 & 23.12 \\
\hline 5 & $16: 38: 18.61$ & $-47: 4: 52.46$ & 0.25 & 0.66 & 0.13 & 0.04 & 0.02 & 23.13 & 22.65 & 22.32 \\
\hline 6 & $16: 38: 18.44$ & $-47: 4: 51.98$ & 0.15 & 0.38 & 0.08 & 0.02 & 0.01 & 23.09 & 22.61 & 22.28 \\
\hline \multicolumn{11}{|c|}{$\mathrm{G} 337.84\left(1.00 \mathrm{e}+07 \mathrm{~cm}^{-3}, 70.0 \mathrm{~K}\right)$} \\
\hline 1 & $16: 40: 26.69$ & $-47: 7: 13.42$ & 2.88 & 135.28 & 66.43 & 21.92 & 10.31 & 24.99 & 24.5 & 24.18 \\
\hline 2 & $16: 40: 26.95$ & $-47: 7: 15.02$ & 4.1 & 73.2 & 35.95 & 11.86 & 5.58 & 24.63 & 24.15 & 23.82 \\
\hline 3 & $16: 40: 26.92$ & $-47: 7: 16.06$ & 4.77 & 85.62 & 42.05 & 13.88 & 6.52 & 24.49 & 24.01 & 23.68 \\
\hline 4 & $16: 40: 26.63$ & $-47: 7: 16.14$ & 3.96 & 66.77 & 32.79 & 10.82 & 5.09 & 24.19 & 23.71 & 23.38 \\
\hline 5 & $16: 40: 26.64$ & $-47: 7: 17.10$ & 5.08 & 64.51 & 31.68 & 10.45 & 4.91 & 24.1 & 23.62 & 23.29 \\
\hline 6 & $16: 40: 26.59$ & $-47: 7: 14.54$ & 3.0 & 47.28 & 23.22 & 7.66 & 3.6 & 24.09 & 23.61 & 23.29 \\
\hline 7 & $16: 40: 26.37$ & $-47: 7: 10.38$ & 0.13 & 0.49 & 0.24 & 0.08 & 0.04 & 23.3 & 22.82 & 22.5 \\
\hline 8 & $16: 40: 27.46$ & $-47: 7: 13.50$ & 0.12 & 0.44 & 0.22 & 0.07 & 0.03 & 23.25 & 22.77 & 22.44 \\
\hline 9 & $16: 40: 26.01$ & $-47: 7: 9.74$ & 0.12 & 0.41 & 0.2 & 0.07 & 0.03 & 23.19 & 22.71 & 22.38 \\
\hline \multicolumn{11}{|c|}{$\mathrm{G} 339.11\left(1.00 \mathrm{e}+07 \mathrm{~cm}^{-3}, 50.0 \mathrm{~K}\right)$} \\
\hline 1 & $16: 42: 59.56$ & $-45: 49: 44.56$ & 32.82 & 189.31 & 104.87 & 34.61 & 16.27 & 23.87 & 23.39 & 23.07 \\
\hline 2 & $16: 43: 0.02$ & $-45: 49: 40.32$ & 11.78 & 52.59 & 29.13 & 9.61 & 4.52 & 23.58 & 23.1 & 22.77 \\
\hline 3 & $16: 43: 0.79$ & $-45: 49: 36.32$ & 0.13 & 0.2 & 0.11 & 0.04 & 0.02 & 22.85 & 22.37 & 22.05 \\
\hline
\end{tabular}

fits for the molecular species are realistic because their partition functions are accurate in the XCLASS database.

The XCLASS routine XCLASSMapFit was used to fit the molecular species mentioned above as well as the H29 $\alpha$ emission. The most robust fitting of a molecule in our XCLASS modelling was that of $\mathrm{CH}_{3} \mathrm{CCH}$, which had five k-ladder transitions in our passband, and therefore also likely gives the best estimate on the physical conditions of the molecular gas surrounding the HII region. This is the case because the properties of the gas surrounding this HII region are good for detecting these transitions of $\mathrm{CH}_{3} \mathrm{CCH}$. Figure B.2 shows the resultant molecular column density, temperature, and velocity offset of the $\mathrm{CH}_{3} \mathrm{CCH}$ within the modelled region of $\mathrm{G} 336.98$ (the same source as shown in Fig. B.1). Where possible, the $\mathrm{CH}_{3} \mathrm{CCH}$ fit properties were used in our analyses (e.g. our Jeans analysis). The regions with the greatest molecular contributions to the $\mathrm{H} 29 \alpha$ spectral region were G336.98 and G337.84, and were therefore the targets for which this analysis was most important.

\section{B.2. Gaussian fitting and spectrum extraction}

The output from XCLASS modelling consisted of a best fit spectrum at each position in the input data cube. This spectrum is a combination of the emission from each modelled species.
As such, a further step of disentangling the $\mathrm{H} 29 \alpha$ emission from the molecular was required. The Gaussians shown in Fig. B.1 represent this subsequent fitting stage after the XCLASS modelling.

These properties were used to constrain subsequent Gaussian fits to the individual components of each species (e.g. the amplitudes, width, and velocities of $\mathrm{CH}_{3} \mathrm{CCH}$ were applied to each of the five components in our spectral window). This was done for each of the fit species (molecular and ionised) to get the individual Gaussian components that make up the combined spectrum in each pixel. As shown in the top panel of Fig. B.1 (taken from a single pixel in the G336.98 map), the combination of fitting $\mathrm{H} 29 \alpha$ (orange Gaussian) and $\mathrm{CH}_{3} \mathrm{CCH}$ (other Gaussians) fits most of the spectral features in this window. The grey filled portion of the spectrum in the top panel shows the profile of the combined Gaussians to highlight that the multi-component fit really is required to account for the ionised and molecular species independently.

With the individual Gaussians computed (using pyspeckit and spectralcube), we can separate the ionised (middle panel) and molecular (bottom panel) components of the emission. The ionised emission spectra (e.g. the original spectrum with the Gaussian fits to the molecular emission removed) were used for the subsequent ionised gas analyses of G336.98 and G337.84. 
P. D. Klaassen et al.: The evolution of young HII regions. I.
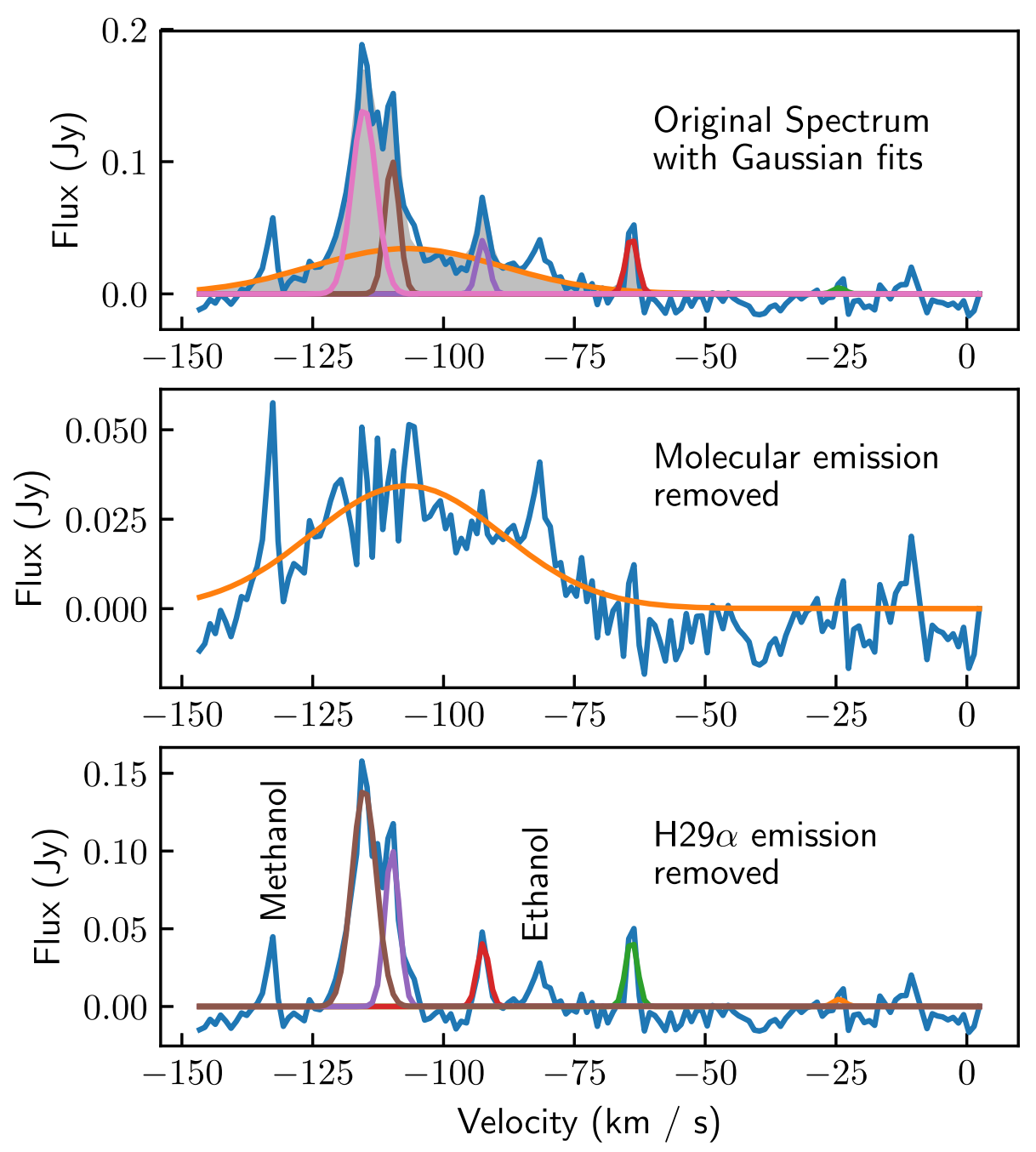

Fig. B.1. $\mathrm{H} 29 \alpha$ and $\mathrm{CH}_{3} \mathrm{CCH}$ Gaussian fits to the emission from G336.98. The blue curve shows the overall emission spectrum, the orange curve shows the Gaussian fit to $\mathrm{H} 29 \alpha$, and the other colour curves show the Gaussian fits to the $\mathrm{CH}_{3} \mathrm{CCH}$ components. In the top panel, the blue curve shows the original spectrum; the grey fill indicates the sum of the Gaussian components at each velocity. In the middle panel, the molecular Gaussian components have been removed, and in the bottom panel, the $\mathrm{H} 29 \alpha$ has been removed, showing how well the individual Gaussian components fit the spectrum. The additional, unfit lines correspond to other species such as methanol and ethanol (at $\sim-130$ and $\sim-80 \mathrm{~km} \mathrm{~s}^{-1}$, respectively), which were not included in the XCLASS fit.
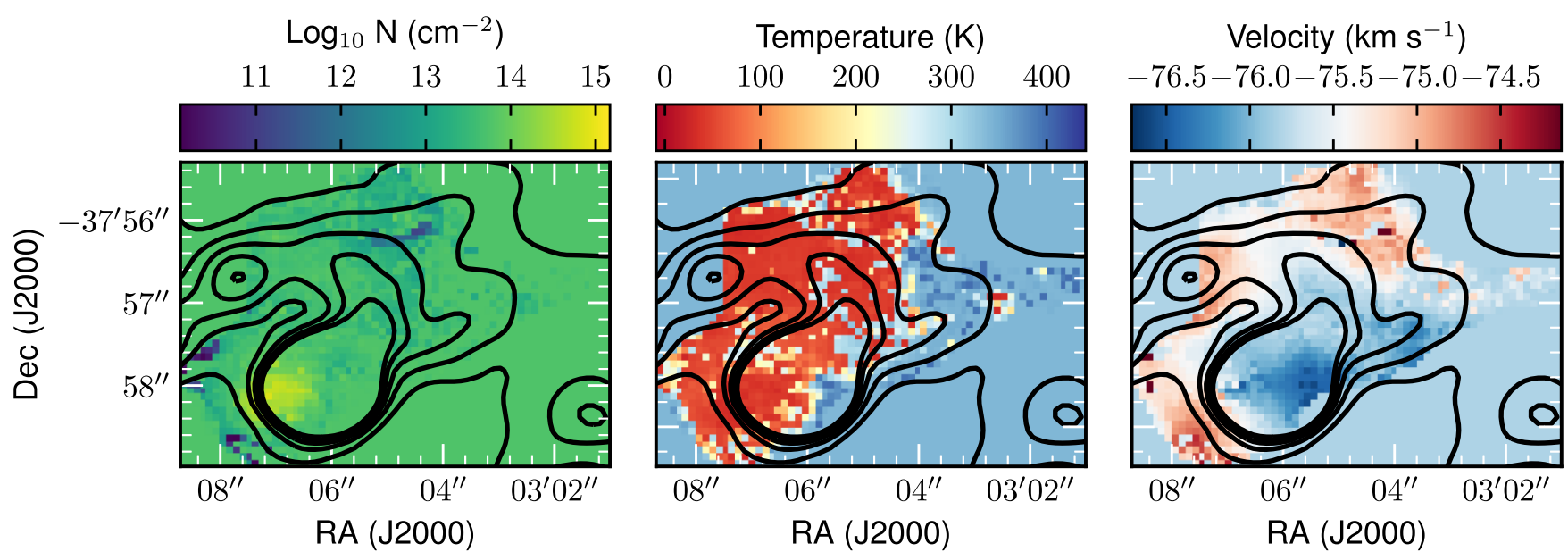

Fig. B.2. Physical properties (e.g. column density, temperature, and velocity structure from left to right) of the $\mathrm{CH}_{3} \mathrm{CCH}_{\text {emission derived through }}$ XCLASS fitting of the five spectral components shown in Fig. B.1 for G336.98. The sharp edge in the temperature and velocity maps indicates the edge of the fitting area rather than a physical jump in parameters. 